3. Maximale Auflösung

Während die Verwerfungsfehler von der Güte der Orientierung der die Linse aufbauenden Kristallite abhängen, kann der chromatische Fehler durch äußere Einflüsse nicht verändert werden. Er ist bedingt durch die Halbwertsbreite der Röntgenspektrallinien ${ }^{6}$.

Zur Abschätzung der maximal erreichbaren Auflösung setzen wir für die Vergrößerung $V$,

${ }^{6}$ Der chromatische Fehler begrenzt daher theoretisch die erreichbare Auflösung. Vergleicht man größenordnungsmäßig nach den angegebenen Fehlerschätzungen die verschiedenen Fehler, so kommt man zum Schluß, daß bei plausiblen Annahmen über Mosaikstruktur Aussicht besteht, die Grenzauflösung zu erreichen. ferner nehmen wir an, daß bei den vorliegenden großen Vergrößerungen $a \approx f$ ist. Wir können schreiben (kleiner Glanzwinkel) :

$$
b=a V, \quad b^{\prime}=\frac{a V}{1 \pm \frac{\Delta \lambda}{\lambda} V} .
$$

$\frac{\Delta \lambda}{\lambda}$ beträgt für $\mathrm{Cu}-K_{\alpha^{-}}$Strahlung ca. $4 \cdot 10^{-4}$.

Der chromatische Fehler kann durch „Abblenden" weiter verkleinert werden, also durch Wahl von Linsen mit kleinerem $r$. Das hat seine praktische Grenze, bedingt durch Linsengröße, Lichtstärke und durch die Linseneigenschaften.

Aus den angegebenen Daten läßt sich eine maximale Auflösung von etwa $1 \mu$ abschätzen.

\title{
Über die Kontraste von Atomen im Elektronenmikroskop
}

\author{
Von Hans BoERsch \\ Aus dem Institut des Recherches Scientifiques in Tettnang (Württ.) \\ (Z. Naturforschg. 2 a, 615-633 [1947]; eingegangen am 18. Juni 1947)
}

\begin{abstract}
In h a lt: 1. Streuabsorption: a) elastische Streuung, b) unelastische Streuung, c) experimentelle Voraussetzungen, d) Kontrast von Einzelatomen, e) Kontraste flächenhafter amorpher Objekte, f) Kontraste von Kristallgittern; Intensität der Kristallgitter-Interferenzen; Kontraste nicht aufgelöster Kristalle. 2. Phasenkontrast: a) Intensität der Amplituden-Gitterinterferenzen, b) Kontraste aufgelöster Kristallgitter, c) Phasenschiebung; Folie; elektrostatische Felder. 3. Filterung: a) Kontrast flächenhafter Objekte, b) Kontrast von Einzelatomen, c) Kontraste aufgelöster Kristallgitter. 4. Linsenfehler. 5. Dunkelfeld: a) nicht freitragende Objektte, b) freitragende Objekte, c) Dunkelfeld-Hellfeld. 6. Zusammenfassung.

Durch diese Untersuchung wird gezeigt, daß schon mittels Streuabsorption Einzelatome höherer Ordnungszahl sichtbar gemacht werden können, wenn es gelingt, das Auflösungsvermögen entsprechend zu steigern. Eine zusätzliche Ausfilterung der unelastisch gestreuten Elektronen bringt besonders bei leichten Atomen eine sehr erwünschte Kontraststeigerung. Eine entscheidende Verbesserung bedeutet aber die Einführung des Phasenkontrastverfahrens in die Elektronen-Optik, so daß nicht nur die leichten Atome der organischen Chemie sichtbar gemacht werden können, sondern auch die Atome im Kristallgitter oder in anderen Atomverbindungen. Auch eine Weiterentwicklung der Technik der Dunkelfeldmethode erscheint sehr lohnend, da sie ebenfalls die leichten Atome der orgạnischen Chemie im festen Körper zu kennzeichnen gestattet.

Die Frage, die den Anlaß zu dieser Untersuchung gab, kann daher dahin beantwortet werden, daß es durchaus sinnvoll ist, das Auflösungsvermögen des Elektronenmikroskops bis zur Auflösung der Atomabstände zu steigern. .
\end{abstract}

$\mathrm{D}^{\mathrm{s}}$ ie Sichtbarmachung der Atome kann als eines der Entwicklungsziele der Elektronenmikroskopie bezeichnet werden. Heute schon gestattet das Elektronenmikroskop, Gegenstände aufzulösen, die nur 5- bis 10-mal größer als ein Atom sind. Es erhebt sich nun die Frage, ob es einen Sinn hat, das Auflösungsvermögen bis zur Auf- lösung der Atomabstände zu steigern, - denn zunächst ist es doch zweifelhaft, ob die Bildkontraste eines so feinen Gebildes, wie es das Atom darstellt, zu einer Sichtbarmachung ausreichen.

In dieser Arbeit sollen daher die Bildkontraste von Atomen untersucht werden. Bei dieser Gelegenheit können auch die Bildkontraste dünnster 
Schichten, deren Atomabstände nicht aufgelöst werden, bestimmt werden, da auch deren Kontraste unmittelbar durch die Vorgänge am Einzelatom verursacht werden.

Damit Bildkontraste entstehen, muß das Objekt eine Änderung der Lichtverteilung in der Bildebene gegenüber der primären Lichtverteilung in der Objektebene heryorrufen. Da die direkte Ausscheidung von Elektronen durch Absorption oder Reflexion im Objekt für die Kontrastbildung im Elektronenmikroskop keine Rolle spielt, ist diese immer ein komplexer Vorgang, der auf einer Beeinflussung der Elektronen im Objekt und im nachfolgenden Abbildungsvorgang beruht. Alle in dieser Untersuchung diskutierten Methoden der Kontrastbildung beruhen darauf, daß im Objekt Elektronen gestreut ${ }^{1}$ werden. In dem Kapitel Streuabsorption, das die heute fast ausschließlich benutzte Methode der Kontrastbildung darstellt, sind daher die Unterlagen für die Streuung zusammengefaßt.

\section{Streuabsorption ${ }^{2}$}

Die Entstehung von Kontrasten durch Streuabsorption wurde experimentell nachgewiesen ${ }^{3}$. Demnach kommt eine Verringerung der Primärintensität durch das Objekt dadurch zustande, daß die vom Objekt gestreuten Elektronen von der Aperturblende des Objektivs abgefangen werden (vergl. Abb.1). Es wurden verschiedentlich Versuche unternommen, die Bildkontraste auf Grund dieser Vorstellung zu berechnen. M a rt o $\mathrm{n}^{4}$ und v. Ardenn e ${ }^{5}$ bestimmten den Kontrast mit Hilfe der für den Fall der Vielfachstreuung gültigen Beziehungen. Diese sind für Einzelatome und extrem dünne Schichten jedoch nicht mehr zutreffend. Hilli e ${ }^{6}$ hat versucht, den Kontrast von Einzelatomen mit Hilfe der Rutherfordschen Streuformel zu gewinnen. Gegen die Anwendung der Rutherfordschen Streuformel ist jedoch einzuwenden, daß die Abschirmung des CoulombFeldes durch die Atomelektronen nicht berücksichtigt wird. Dieser Einfluß wird aber gerade für kleine Ablenkwinkel, also für kleine Objektivöffnungen, die heute wegen der geometrisch-

1 Methoden, die allein die Beschleunigung oder Verzögerung der Elektronen im Objekt durch ein konstantes inneres Potential zur Kontrastbildung ausnutzen, sind für das Elektronenmikroskop bisher nicht bekannt geworden. Man könnte hier an ähnliche Anordnungen wie die Interferometer der Lichtoptik denken. optischen Linsenfehler noch notwendig sind, von Bedeutung. Infolgedessen liefert die Methode von Hillier zu starke Kontraste. Außerdem bezieht sich der Ansatz von Hillier nur auf die elastische Streuung, während der Einfluß der unelastischen nicht in Rechnung gesetzt wird.

Es soll daher versucht werden, den Einfluß der Kernabschirmung durch die Atomelektronen und den Einfluß der unelastischen Streuung auf die Streuabsorption zu erfassen.

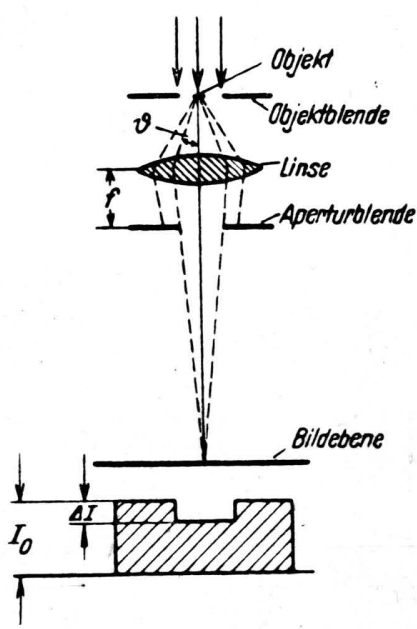

Abb. 1. Kontrast durch Streuabsorption.

a) Elastische Streung

Zur Bestimmung der elastischen Streuung kommt das Hartreesche Atommodell (Methode des „self consistent field“) und das Atommodell von Thomas-Fermi in Betracht. Zwar würde das Hartree-Modell die exaktesten Unterlagen liefern, jedoch besitzt es den Nachteil, daß individuelle Rechnungen für-jedes einzelne Atom notwendig sind. Daher wurde den folgenden Untersuchungen das Thomas-Fermi-Atom, dessen Potential-Verteilung mit Hilfe einer universellen Funktion darstellbar ist, zugrunde gelegt. Selbstverständlich sind auf die darauf fußenden Beziehungen alle für das Thomas-Fermi-Atom geltenden Einschränkungen anzuwenden. Die errechneten Kontraste beziehen sich also auf Atome höherer Ord-

2 H. B o e r s ch, Mh. Chem. 1946, 86 u. 163.

3 H. B o e rs c h, Ann. Physik 26,631 [1936] ; 27, 75 [1936].

4 L. Marton, Physica 3, 959 [1936].

5 M. v. A r d enn e, Elektronenübermikroskopie, S. 60 , Berlin 1940 .

6 J. H i ll i e r, Physic. Rev. 60, 743 [1941]. 
nungszahlen. Ferner werden die an den äußeren Bezirken des Thomas-Fermi-Atoms gestreuten Elektronen - also die Streuelektronen geringen Ablenkwinkels - Abweichungen gegenüber denen des Realatoms aufweisen.

Die Zahl der am Thomas-Fermi-Atom elastisch gestreuten Elektronen beträgt:

$$
N_{e} d \vartheta=N_{0}\left(\frac{Z e^{2}}{2 m v^{2}}\right)^{2} \frac{(1-f)^{2}}{\sin ^{4} \vartheta / 2} 2 \pi \sin \vartheta d \vartheta
$$

oder

$$
N_{e} d \xi=N_{0} x \hat{\lambda}^{2} Z^{4 / 3} \frac{(1-f)^{2}}{\xi^{4}} \xi d \xi .
$$

Hierin ist $\frac{(1-f)^{2}}{\xi^{t}}$ eine von $\mathrm{B}$ eth $\mathrm{e}^{7}$ tabellierte Funktion mit $\xi=\frac{\sin \vartheta / 2}{\lambda Z^{1 / 3}} ; \dot{\varkappa}=2 \pi \cdot 10^{-32} \frac{e^{4} m^{2}}{\iota^{4}}$ $=1,52 \cdot 10^{-18} ; N_{0}$ die Zahl der Primärelektronen pro $\mathrm{cm}^{2} ; \vartheta$ der Streuwinkel; $\lambda$ die Wellenlänge in A und $Z$ die Ordnungszahl.

Wenn der Öffnungswinkel des Objektivs mit $\vartheta$ identifiziert wird (vergl. Abb.1), wird die Zahl der von der Aperturblende abgefangenen, also der elastisch streuabsorbierten Elektronen:

$$
N_{e}=\int_{\xi}^{\xi\left(180^{\circ}\right)} N_{e} d \xi
$$

Da die Tabelle von $\frac{(1-f)^{2}}{\xi^{4}}$ nur von $\xi=0$ bis $\xi=0,5$ reicht, wurde anschließend von $\xi=0,5$ bis $\xi\left(180^{\circ}\right) \sim \infty$ die Rutherfordsche Streuformel benutzt, die bei $\xi=0,5$ nur noch geringe $A b$ weichungen (10\%) von den Betheschen Werten für das Thomas-Fermi-Atom aufweist:

$N_{e}=$

$N_{0} \varkappa \lambda^{2} Z^{4 / 3}\left(\int_{\xi}^{0,5} \frac{(1-f)^{2}}{\xi^{4}} \xi d \xi+\int_{0,5}^{\infty} \frac{1}{\xi^{3}} d \xi\right)$.

Das erste Integral wurde mit Hilfe der Simpsonschen Regel numerisch bestimmt; das zweite ergibt den Wert 2.

Die Zahl der vom Thomas-Fermi-Atom elastisch streuabsorbierten Elektronen ist in Abb. 2 als Funktion von $\xi$ dargestellt. In der Nähe des Streuwinkels $\vartheta=0$ beträgt die Zahl der streuabsorbier-

7 H. B ethe, Ann. Physik 5, 325 [1930]. ten Elektronen, also die Gesamtzahl der am Thomas-Fermi-Atom elastisch gestreuten Elektronen:

$$
\lim _{\xi=0}{ }_{a} N_{e}={ }_{a} N_{e}(0)=N_{0} \cdot 41,5 \cdot \varkappa \lambda^{2} Z^{4 / 3} .
$$

In Abb. 2 sind zum Vergleich auch die nach der Rutherfordschen Beziehung streuabsorbierten Elektronen angegeben. Das Streuvermögen des Thomas-Fermi-Atoms nimmt, wie erwartet, gegenüber der Rutherfordschen Streuformel mit abnehmendem Streuwinkel und steigender Ordnungszahl (stärkere Kernabschirmung) ab.

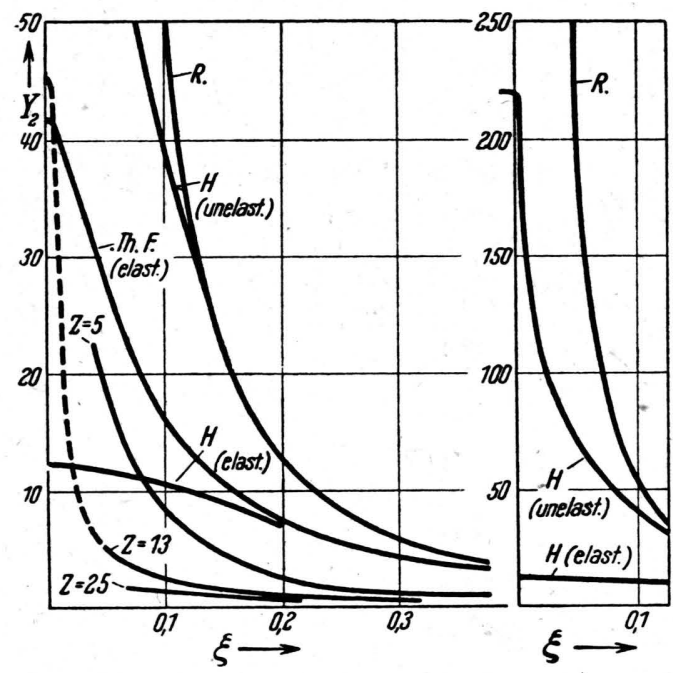

Abb. 2. Elastische Streuabsorption des Rutherford- (R.), Thomas-Fermi- (Th.-F.) und H-Atoms. Unelastische Streuabsorption des Thomas-Fermi- $(Z=5,13,25)$ und H-Atoms. $Y_{2}={ }_{a} N / N_{0} \varkappa \lambda^{2} Z^{4 / 3} ; \xi=\sin \frac{\vartheta}{2} / \lambda Z^{1 / 3}$; $\varkappa=1,52 \cdot 10^{-18} ; \lambda$ in $\AA$.

Für den Wasserstoff lassen sich nach der erwähnten Arbeit von Bethe exakte Angaben über die elastische Streuung machen, so daß hier ein Vergleich mit dem Resultat der Thomas-FermiMethode möglich ist, die als statistische Methode für das Ein-Elektronen-Problem des Wasserstoffs die ungenauesten Resultate liefert. Obwohl der Wasserstoff wegen seiner geringen Kernladungszahl für die Kontrastbildung im Elektronenmikroskop im allgemeinen keine besondere Rolle spielt, beansprucht er doch als klarer und bekannter Grenzfall besonderes Interesse.

Die Zahl der am Wasserstoffatom elastisch gestreuten Elektronen beträgt nach $\mathrm{B}$ e the : 
$N_{e}^{\mathrm{H}} d \xi=N_{0} \varkappa \lambda^{2} \frac{1}{\xi^{3}}\left\{1-\frac{1}{\left(1+\xi^{2} \cdot 10^{-16} / 2 \pi x\right)^{2}}\right\}^{2}$.

Für große Winkel geht diese Beziehung in die Rutherfordsche Streuformel über.

Die Zahl der streuabsorbierten Elektronen ergibt sich aus (6) wiederum durch Integration zu:

$$
{ }_{a} N_{e}^{\mathrm{H}}=\int_{\xi}^{\xi\left(180^{\circ}\right)} N_{e}^{\mathrm{H}} d \xi
$$

Dieses Integral, das wieder durch Anwendung der Simpsonschen Regel im Bereich $\xi=0$ bis $\xi=0,5$ und durch Integration der Rutherfordschen Streuformel im Bereich von $\xi=0,5$ bis $\xi=\infty$ gewonnen wurde, ist u. a. in Abb. 2 dargestellt. Aus dieser Abbildung geht hervor, daß die exakte Lösung des Ein-Elektronen-Problems für die elastische Streuabsorption maximal. um etwa den Faktor 3 von den Werten abweicht, die die statistische Näherungsmethode von ThomasFermi liefert. Die Abweichungen betragen also. selbst in dem extrem ungünstigen Fall des Wasserstoffs noch keine Größenordnung. Bei Atomen mit höheren Ordnungszahlen ist daher ein rasches Angleichen der nach der Thomas-FermiMethode gewonnenen an die tatsächlichen Werte zu erwarten.

b) Unelastische Streuung

Für die unelastische Streuung liegen leider nicht so vollständige Unterlagen wie für die elastische vor. Nach Wissen des Verfassers existiert nur eine Abschätzung von $\mathrm{He}$ is e $\mathrm{n} b$ erg ${ }^{8}$, die auf einer Erweiterung des Thomas-Fermi-Ansatzes für die Elektronendichte im Atom beruht, sowie eine Berechnung der unelastischen Streu- ung des Wasserstoffs (Ein-Elektronen-Problem) in der Arbeit von Bethe ${ }^{7}$ auf Grund der Bornschen Stoßmethode.

Nach der von Bewilogua ${ }^{9}$ erfolgten Auswertung der Heisenbergschen Abschätzung beträgt die Zahl der unelastisch am Thomas-FermiAtom gestreuten Elektronen:

$$
N_{u} d \xi=N_{0} x \lambda^{2} Z^{4 / 3} \frac{S(v)}{Z \xi^{3}} d \xi .
$$

Hierin ist: $S(v)$ eine von Bewilogua tabeldierte Funktion mit $v=\frac{2,213}{Z^{1 / 3}} \xi$. Selbstverständlich sind auch auf diese Beziehung alle für das Thomas-Fermi-Atom geltenden und schon erwähnten Einschränkungen anzuwenden. Sie gilt also nur für Atome höherer Ordnungszahl. Ferner muß die Streuverteilung des Thomas-FermiAtoms bei geringen Streuwinkeln Abweichungen gegenüber der des Realatoms aufweisen. Die Abweichungen sind für die Näherungsmethode Heisenbergs so stark, daß der Grenzwert für den Streuwinkel $\vartheta=0$ nicht angegeben werden kann und die Funktion $S(v)$ an der Stelle $v=0,05$ abgebrochen wird.

Die Zahl der unelastisch streuabsorbierten Elektronen wird nach Integration der Gl. (8) :

$$
{ }_{a} N_{u}=\int_{\xi}^{\xi\left(180^{\circ}\right)} N_{u} d \xi
$$

Da die Tabelle $S(v)$ nur bis zu dem Maximalwert $v=1,1$ reicht und dort $S(v)$ nur noch um 4\% von dem Grenzwert 1 abweicht, wurde für die Integration anschließend an $v=1,1$ bis $v$ $\left(\vartheta=180^{\circ}\right) \sim \infty$ wieder die mit dem Faktor $1 / Z$ versehene Rutherfordsche Streuformel benützt:

$$
{ }_{a} N_{u}=N_{0} x \lambda^{2} Z^{4 / 3}\left(\int_{\xi}^{\xi(v=1,1)} \frac{S(v)}{Z \xi^{3}} d \xi+\frac{1}{Z} \int_{\xi(v=1,1)}^{\infty} \frac{1}{\xi^{3}} d \xi\right) .
$$

Das erste Integral wurde mit Hilfe der Simpsonschen Regel bestimmt, das zweite ergibt den Wert $2 / Z^{5 / 3}$. Die Zahl der von einem Thomas-Fermi-

"W. He is e n be r g, Physik. Z. 32, 737 [1931].

${ }^{9}$ L. B ewil og u a, Physik. Z. 32, 740 [1931].
Atom unelastisch streuabsorbierten Elektronen ist in Abb. 2 dargestellt.

$\mathrm{Zu}$ Aussagen über die unelastische GesamtStreuung (Streuabsorption bei $\xi=0$ ) kann man erst durch Kombination der Messungen von 
Len a rd und $\mathrm{R} u$ them an $\mathrm{n}$ über Geschwindigkeitsverluste beim Durchgang von Elektronen durch Folien (vorläufig nur in dem Spezialfall des Al) gelangen. Dié Reșultate von Lenard über den wahrscheinlichsten Geschwindigkeitsverlust sind in Form des Widding to n schen Gesetzes ${ }^{\mathbf{1 0}}$ darstellbar. In etwas anderer Schreibweise und extrapoliert auf ein Atom mit dem Atomgewicht $M$ pro $\mathrm{cm}^{2}$ bestrahlter Fläche beträgt der wahrscheinlichste Geschwindigkeitsverlust in e-Volt:

$$
\overline{\Delta U}=1,18 \cdot 10^{-15} \lambda^{2} M .
$$

Nach den Beobachtungen von $\mathrm{Ruthemann}{ }^{11}$ finden nun in dünnsten Schichten diskrete Geschwindigkeitsverluste statt (bei Aluminium-Folien: $\Delta U=\mathbf{1 5 , 1} \mathrm{e}-\mathrm{Volt})$. Da der wahrscheinlichste Geschwindigkeitsverlust wenigstens angenähert mit dem mittleren übereinstimmt, wird:

$$
{ }_{a} N_{u}(0)=N_{0} \cdot 1,18 \cdot 10^{-15} \frac{\lambda^{2} M}{\Delta U} .
$$

Da besonders bei größeren Streuwinkeln auch höhere Energieverluste auftreten, stellt der in (12) angegebene Wert sicher einen Maximalwert dar.

Der so erhaltene Wert der unelastischen Gesamtstreuung ist für den Fall des Aluminiums ( $Z=13$ ) in Abb. 2 eingezeichnet und mit der unelastischen Streuabsorption des Thomas-FermiAtoms durch eine punktierte Linie verbunden. Es sei darauf hingewiesen, daß die Streuabsorptionskurven sich auf „freie“ Atome beziehen, dagegen der "Meßpunkt" von $Z=13$ bei $\xi=0$ auf ein „,im festen Körper gebundenes" Atom.

Bemerkenswert ist, daß auch hier ein außerordentlich steiler Anstieg der unelastischen Streuung bei kleinstem Streuwinkel eintritt, daß aber die unelastische Gesamtstreuung nur die gleiche Größenordnung wie die elastische besitzt, im Gegensatz zu den Verhältnissen des Wasserstoff-' Atoms. Es scheint also auch das Verhältnis der elastischen zur unelastischen Gesamtstreuung mit der Ordnungszahl zuzunehmen.

Für den Wasserstoff liegen auf Grund der Arbeit von $\mathrm{B}$ et $\mathrm{h} \mathrm{e}^{7}$ ebenfalls eingehendere Unterlagen über die unelastische Streuung vor. Die

10 Vergl. z. B. W. B oth e, Handbuch d. Physik XXII, S. 32, Berlin 1933.

11 G. Ruthem an n, Naturwiss. 29, 648 [1941].
Zahl der am Wasserstoff unelastisch gestreuten Elektronen läßt sich demnach durch Näherungen, die in verschiedenen Bereichen gültig sind, ausdrücken :

a. Für den Grenzfall $\vartheta=0$ gilt:

$$
\lim _{\vartheta=0} N_{u}^{\mathrm{H}} d \xi=N_{0} \frac{2 \cdot 10^{-48}}{\pi^{3} \varkappa^{2}} \xi d \xi .
$$

b. Für $\frac{\Delta U}{U}<\vartheta<\sqrt{\frac{\Delta U}{U}}$ (wobei $\Delta U$ die Ionisierungsspannung des Wasserstoffs und $U$ die Voltgeschwindigkeit der Primärelektronen bedeuten) gilt:

$$
N_{u}^{\mathrm{H}} d \xi=\dot{N}_{0} \frac{2}{\pi} \cdot 10^{-16} \frac{\lambda^{2}}{\xi} d \xi .
$$

c. Für größere Winkel $\vartheta>\sqrt{\frac{\Delta U}{U}}$ gilt:

$$
\begin{aligned}
N_{u}^{\mathrm{H}} d \xi & =N_{0} \cdot 8 \cdot 10^{32} \varkappa \lambda^{4} \frac{4-3 \sin ^{2} 2 \vartheta}{\sin ^{3} 2 \vartheta} \\
& \approx N_{0} \varkappa \lambda^{2} \frac{d \xi}{\xi^{3}} .
\end{aligned}
$$

Die Funktion (13c) stimmt für kleine Winkel zunächst mit der Rutherfordschen Streuformel überein. Ihr Wiederanstieg bei größeren Streuwinkeln ist durch Mitzählung der Sekundärelektronen niederer Geschwindigkeit bedingt, die auf die Kontrastbildung ohne Einfluß sind. Wie angedeutet, kann daher die Beziehung (13c) durch die Rutherfordsche Streufunktion ersetzt werden, da wegen des starken Intensitätsabfalles ohnehin nur Streuelektronen geringen Ablenkwinkels für die Integration von Bedeutung sind.

Die gesamte unelastische Streuverteilung wurde aus den Funktionen (13a, b, c) durch Zusammensetzen gewonnen. Die Schnittpunkte

$$
\xi_{a b}=\pi 10^{16} \varkappa \lambda \text { und } \xi_{b c}=10^{8} \sqrt{x \frac{\pi}{2}}
$$

der Funktionen (13a,b) bzw. (13b,c) stimmen mit den oben angegebenen Gültigkeitsgrenzen der Funktion (13b) überein, so daß zumindest eine Überbeanspruchung dieser Näherung vermieden ist.

Aus der so zusammengesetzten Streufunktion wurde durch Integration der Einzelfunktion die unelastische Streuabsorption des Wasserstoffs ermittelt: 


$$
\begin{aligned}
0 \leq \xi \leq \xi_{a b} \quad{ }_{a} N_{u}^{\mathrm{H}}=N_{0} \varkappa \lambda^{2}\left(\frac{10^{16}}{\pi \varkappa}-\frac{\xi^{2}}{\pi^{3} 10^{48} \varkappa^{3} \lambda^{2}}\right)+{ }_{a} N_{u}^{\mathrm{H}}\left(\xi_{a b}\right), \\
\xi_{a b} \leq \xi \leq \xi_{b c} \quad{ }_{a} N_{u}^{\mathrm{H}}=N_{0} \frac{2}{\pi} 10^{16} \lambda^{2} \ln \frac{10^{8}}{\xi} \sqrt{\varkappa \frac{\pi}{2}}+{ }_{a} N_{u}^{\mathrm{H}}\left(\xi_{b c}\right), \\
\xi_{b c} \leq \xi<\infty \quad{ }_{a} N_{u}^{\mathrm{H}}=N_{0} \frac{\varkappa}{2} \frac{\lambda^{2}}{\xi^{2}} .
\end{aligned}
$$

In Abb. 2 ist u. a. diese unelastische Streuabsorption des Wasserstoffs für die Wellenlänge $\lambda=0,05 \AA$ dargestellt. Der Fehler, der sich durch das übergangslose Aneinandersetzen und die Extrapolation der drei Einzelfunktionen ergibt, dürfte $\mathrm{zu}$ vernachlässigen sein, da die so erhaltene unelastische Gesamtstreuabsorption

$$
{ }_{u} N_{a}^{\mathrm{H}}(0)=N_{0} \frac{\lambda^{2}}{\pi} \ln \frac{1,18 \cdot 10^{-32}}{\varkappa \lambda^{2}}
$$

für den Streuwinkel $\vartheta=0$ bis auf $1,4 \%$ für $\lambda=0,05 \AA$ übereinstimmt mit dem von $\mathrm{B}$ eth e auf anderem Weg erhaltenen. „unelastischen Gesamtwirkungsquerschnitt".

Das Verhältnis der elastischen Streuabsorption zur unelastischen ist bei größeren Objektivöffnungen $Z: 1$. Bei kleinen Objektivöffnungen ist das rapide Anwachsen der unelastischen Streuabsorption gegenüber der elastischen bemerkenswert, jedoch scheint auch hier, wie der Vergleich der Streukurven des Thomas-Fermi-Atoms und der Vergleich der Gesamtstreuung des Wasserstoffs und des Aluminiums zeigt, das Verhältnis der elastischen zur unelastischen Streuabsorption mit wachsender Ordnungszahl zuzunehmen.

c) Experimentelle Voraussetzungen

Zur Abschätzung der Kontraste sind, wie sich aus dem vorhergehenden Abschnitt ergibt, An-

12 In dieser Untersuchung taucht der Begriff des Auflösungsvermögens in verschiedener Form und mit verschiedenem Inhalt auf. Der Kürze halber seien folgende Definitionen gegeben:

Ideales Auflösungsvermögen bedeutet, daß der Idealzustand des Auflösungsvermögens für eine bestimmte Objektivapertur erreicht ist. Es ergibt sich nach der Beugungstheorie des Gitters aus der Objektivapertur $\vartheta$ und der Wellenlänge $\lambda$ zu $d=\lambda / \sin \vartheta$.

Prinzipielles Auflösungsvermögen bedeutet, daß das optische System prinzipiell Abstände der Größe $d=\lambda / \sin \vartheta$ auflösen könnte, wenn nicht andere $\mathrm{Ab}$ bildungsfehler die Erreichung dieses Idealzustandes verhindern würden. Das prinzipielle Auflösungsver- gaben über die Objektivöffnungen, Wellenlängen und die Größe der nachweisbaren Elektronenkontraste erforderlich.

Von gewissen Ausnahmen abgesehen, benutzt man heute Aperturblenden von etwa $0,05 \mathrm{~mm} \varnothing$. Die Brennweiten betragen beim elektrostatischen Mikroskop etwa $5 \mathrm{~mm}$, beim magnetischen etwa $2 \mathrm{~mm}$. Hieraus ergeben sich Objektivöffnungen von $5 \cdot 10^{-3}$ bzw. $1,2 \cdot 10^{-2}$. Da im elektrostatischen Mikroskop Wellenlängen von etwa $0,05 \AA$ ( $60 \mathrm{eKV})$ und im magnetischen Mikroskop Wellenlängen von etwa $0,037 \AA$ (100 eKV) gebräuchlich sind, so sind die Kontraste für Werte von $\xi=0,05 / Z^{1 / 3}$ bzw. $\xi=0,16 / Z^{1 / 3}$ zu bestimmen. Im Durchschnițt dürfte der letzte Wert gelten, der einem prinzipiellen Auflösungsvermögen ${ }^{12}$ von $d=3 \AA$ entspricht, das in der Praxis wegen der Abbildungsfehler bisher nicht erreicht worden ist.

Obwohl die in Abb. 2 dargestellten Funktionen der unelastischen Streuabsorption im allgemeinen zu früh abbrechen, um ein vollkommenes Bild der unelastischen Streuabsorption $\mathrm{zu}$ vermitteln, ist der Abbildung für den Wert von $\xi=0,16 / Z^{1 / 3}$ und bei höheren Ordnungszahlen $\mathrm{zu}$ entnehmen, daß die unelastische Streuabsorption neben der elastischen nur eine im allgemeinen $\mathrm{zu}$ vernachlässigende Korrektur bedeutet. Für eine Abschätzung der Kontraste durch Streuabsorption genügt also unter den angegebenen Voraussetzungen im allgemeinen die Berücksichtigung allein der elastischen Streuabsorption.

mögen ist also nur ein Maß für das Verhältnis Wellenlänge zu Objektivöffnung.

Theoretisches Auflösungsvermögen ergibt sich aus der Úberlagerung aller theoretisch erfaßten Abbildungsfehler, wie Beugungsfehler, sphärische und chromatische Aberration, Astigmatismus, Koma usw.

Optimales Auflösungsvermögen ergibt sich aus der optimalen Überlagerung aller theoretisch erfaßten Abbildungsfehler.

Praktisches Auflösungsvermögen bedeutet das im Experiment tatsächlich erreichte Auflösungsvermögen.

Großes Auflösungsvermögen bedeutet kleine aufgelöste Objektabstände und umgekehrt. 
Férner wird angenommen, daß Elektronen-Kontraste $: \Delta I / I_{0}=I_{0}-I_{1} / I_{0}=0,1$ gerade noch photographisch nachweisbar sind. In dieser Annahme ist schon ein gewisser Sicherheitsfaktor enthalten, da in einer vorangegangenen Arbeit ${ }^{2}$ die Kontrasterkennbarkeit für Elektronen-Kontraste auf Grund der Messungen zu $\Delta I / I_{0}=0,04$ bestimmt worden war.

\section{d) Kontrast von Einzelatomen}

Zur Bestimmung des Kontrastes von Einzelatomen, die in der Gegenstandsebene weit auseinanderliegen, möge angenommen werden, daß das Atom durch sein Beugungsfehlerscheibchen dargestellt wird. Es wird also angenommen, daß der Einfluß der Abbildungsfehler auf die Abbildung $\mathrm{zu}$ vernachlässigen ist, was ohne wesentliche Einbuße an praktischem Auflösungsvermögen ${ }^{12}$ durch entsprechende Verringerung der Objektivapertur $\mathrm{zu}$ erreichen ist. Ferner wird vorausgesetzt, daß das Atom klein gegen sein Bild ist.

Die Intensitätsverteilung im Bild ist also durch die Intensitätsverteilung des Beugungsfehlerscheibchens darstellbar, die durch die Funktion

$$
\Delta I=C\left(\frac{I_{1}(x)}{x}\right)^{2}
$$

gegeben ist. Hierbei stellt $I_{1}(x)$ die erste Besselfunktion mit $x=2 \pi r \frac{\sin \vartheta}{\lambda}=4 \pi 10^{8} r Z^{1 / 3} \xi$ und $r$, der Entfernung des Aufpunktes in der Bildebene, dar. $C$ bedeutet eine Konstante, die im folgenden bestimmt werden soll, indem die Gesamtintensität der Beugungsfigur der Zahl der streuabsorbierten Elektronen gleichgesetzt wird:

$$
a^{N}=C \int_{0}^{+\infty}\left(\frac{I_{1}(x)}{x}\right)^{2} 2 \pi r d r=C \frac{\lambda^{2}}{4 \pi \sin ^{2} \vartheta} .
$$

Da (für $x=0$ ) die maximale Intensität des Beugungsfehlerscheibchens $\Delta I(0)=C / 4$ wird, ergibt sich aus (16) und (17) der maximale Elektronenkontrast im Zentrum des Beugungsfehlerscheibchens zu:

$$
\frac{\Delta I(0)}{I_{0}}=4 \pi 10^{16} Z^{2 / 3} \xi^{2} \frac{a^{N}}{N_{0}},
$$

während in einer vorangegangenen Arbeit $^{2}$ dieser Wert durch einen um den Faktor 0,405 kleineren Mittelwert ersetzt wurde. Ausdrücklich sei je- doch darauf hingewiesen, daß beide Werte nur einen Anhaltspunkt über die zu erwartenden Kontraste liefern, denn der obige Ansatz über die Intensitätsverteilung im Beugungsfehlerscheibchen beruht auf der Voraussetzung, daß der streuende Körper eine Strahlungscharakteristik liefert, die für das Atom nur angenähert zutrifft.

In Abb. 3 ist die Funktion (18) für den Fall der elastischen Streuabsorption dargestellt. Da un-

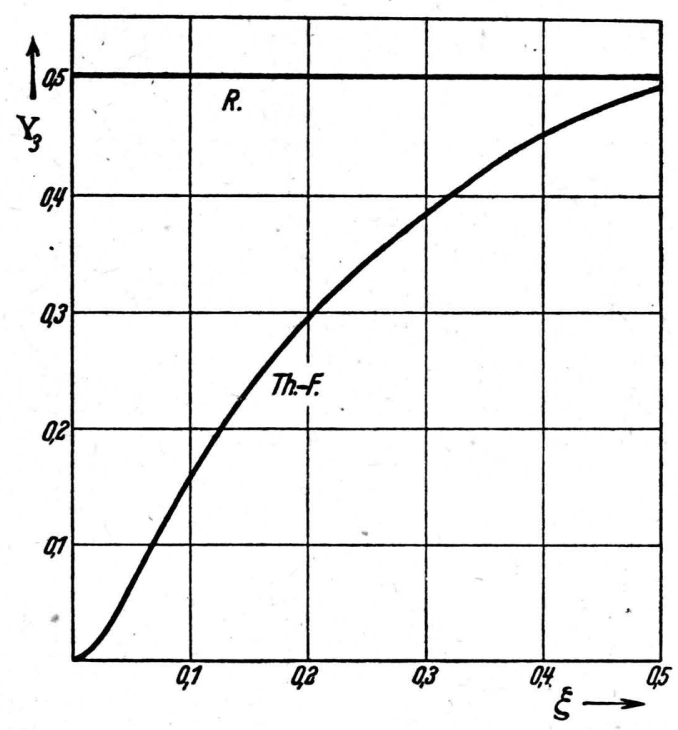

Abb. 3. Elektronenkontrast des Einzelatoms durch elastische Streuabsorption. R. = Rutherford, Th.-F. = Thomas-Fermi. $Y_{3}=\Delta I / I_{0} 4 \pi 10^{16} * \lambda^{2} Z^{2} ; \xi=\frac{\sin \vartheta / 2}{\lambda Z^{1 / 3}}$; $\varkappa=1,52 \cdot 10^{-18} ; \lambda$ in $\AA$.

elastische Streuabsorption, insbesondere für schwere Atome und größere Ảperturen nur eine Korrektion der elastischen bedeuten dürfte (vergl. Tab. 1), ist sie nicht berücksichtigt. Für das Rutherford-Atom ergibt sich der Kontrast als unabhängig von der Objektivapertur. Dagegen nimmt er bei dem Thomas-Fermi-Atom von Null an mit steigender Apertur zu, um schließlich dem Grenzwert des Rutherford-Atoms zuzustreben.

Wenn das ideale Auflösungsvermögen durch Verringerung der Objektivapertur mit dem heute ${ }^{13}$ erreichten praktischen Auflösungsvermögen von $12 \AA$ in Übereinstimmung gebracht wird, ergibt sich aus (18), daß bei $100 \mathrm{eKV}$ erst Atome der

13 Die Auflösung von 12 A wurde durch den Verfasser im November 1944 mit dem Siemens-Übermikroskop erzielt. 
Ordnungszahl $Z \geqq 560$ nachgewiesen werden können. Unter diesen Voraussetzungen ist also der Nachweis der Einzelatome nicht möglich. Dagegen sollten bei einem idealen Auflösungsvermögen von $3 \AA$ A , wie es zur Trennung der Atomabstände mindestens notwendig wäre, und einer Wellenlänge $\lambda=0,05 \AA$ Atome mit Ordnungszahlen $Z \geqq 64$ erkennbar werden. Unabhängig von der Größe des idealen Auflösungsvermögens wäre dagegen das Rutherford-Atom schon von einer Ordnungszahl $Z \geqq 28$ nachweisbar. Auch in diesen Beispielen wurde die unelastische Streuabsorption in Anbetracht der relativ hohen Ordnungszahlen nicht berücksichtigt (vgl. auch Tab.1).

e) Kontrast flächenhafter, amorpher $\mathrm{Objekte}$

Der Kontrast flächenhafter Objekte, deren Atome im Mikroskop nicht aufgelöst werden, ergibt sich aus der Zahl der streuabsorbierten Elektronen zu:

$$
\frac{\Delta I}{I_{0}}=n \frac{a^{N}}{N_{0}},
$$

wenn $\boldsymbol{n}$ die Zahl der Atome pro Quadratzentimeter bedeutet. Hierbei wird vorausgesetzt, daß sich die Streuung der Einzelatome gegenseitig nicht beeinflußt.

Unter diesen Voraussetzungen wurden in Tab. 1 die geringsten Schichtdicken (Grenzdicken) ermittelt, die im Elektronenmikroskop mittels Streuabsorption noch nachgewiesen werden können. Hierfür wurde ein Atomabstand von $3 \mathrm{~A}$ bei dichtester Kugelpackung zugrunde gelegt.

Experimentell sind Kontraste von etwa $100 \AA$ starken Zaponfolien feststellbar $\left(\vartheta=10^{-2}\right.$, $\lambda=0,037 \AA$ A), während sich für eine Bruttoformel $\mathrm{C}_{12} \mathrm{H}_{18} \mathrm{O}_{10}$ und das spezifische Gewicht 1,3 aus der elastischen Streuabsorption eine Dicke von 160 A und unter Berücksichtigung der unelastischen Streuabsorption eine Dicke von 102 A ergibt.

\begin{tabular}{|c|c|c|c|c|}
\hline$Z$ & 1 & 5 & 25 & 90 \\
\hline $\begin{array}{l}\text { Elastische Streuabsorption }(\AA) \\
\begin{array}{c}\text { Elastische und unelastische } \\
\text { Streuabsorption }(\AA)\end{array}\end{array}$ & 9600 & 640 & 46 & 7 \\
\hline
\end{tabular}

Tab. 1. Grenzdicken amorpher Schichten (in A) bei einer Elektronen-Energie von 100 eKV und einem prinzipiellen Auflösungsvermögen von 3 A für verschiedene Ordnungszahlen $Z$.
Selbstverständlich ist diese genaue Übereinstimmung zufälliger Natur.

f) Kontraste von Kristallgittern ${ }^{14}$

In dem vorangegangenen Abschnitt waren Kontraste dünner Materieschichten angegeben worden, unter der Voraussetzung, daß sich die Streuung der Einzelatome gegenseitig nicht beeinflußt. Für die elastische Streuung gilt dies streng nur für den Fall der idealen Unordnung, also beispielsweise für das mono-atomare Gas. Schon im mehratomigen Gas, in der Flüssigkeit und im reellen ,amorphen“ Körper (z.B. Gläser) herrscht jedoch ein Ordnungszustand, der zu einer - manchmal allerdings nur geringfügigen Änderung der Streuverteilung gegenüber der des Einzelatoms führt. Am stärksten prägt sich die gegenseitige Beeinflussung der Streuung der Einzelatome im Zustand der höchsten Ordnung, nämlich in der Gitteranordnung des Kristalls, aus. Dieser Zustand führt bekanntlich zu einer Konzentration der Streuintensität in die Maxima der Kristallgitterinterferenzen. In diesem Abschnitt sollen daher die Bildkontraste dünner Kristallschichten ermittelt werden, unter der Voraussetzung, daß die Gitterstruktur nicht aufgelöst wird.

Hierfür ist die Kenntnis der Intensität notwendig, die in die einzelnen Interferenzmaxima gestreut wird. Da es sich um die Bestimmung der Grenze der Kontrasterkennbarkeit handelt, kann vorausgesetzt werden, daß die Änderung der Primärstrahlintensität durch die Streuvorgänge noch ohne wesentlichen Einfluß auf die Interferenzintensität ist, so daß die kinematische Theorie von v. La ue ausreicht.

Intensität der Kristallgitterinterferenzen. Für ein rhombisches Kristallgitter mit der Achse $a_{3}$ parallel zur Mikroskopachse und den Achsen $a_{1}$ und $a_{2}$ parallel zur Objektivebene wird die Streuintensität nach v. La ue:

$$
\begin{aligned}
& N_{e}^{k}= \\
& N_{0} \Phi^{2} S^{2} \frac{\sin ^{2} N_{1} A_{1}}{\sin ^{2} A_{1}} \cdot \frac{\sin ^{2} N_{2} A_{2}}{\sin ^{2} A_{2}} \cdot \frac{\sin ^{2} N_{3} A_{3}}{\sin ^{2} A_{3}} .
\end{aligned}
$$

Hierin ist $N_{0}$ die Primärintensität, $\Phi^{2}$ der Atomfaktor, $S^{2}$ der Strukturfaktor, $N_{i}$ die Zahl der ${ }_{14}$ H. B o e r s c h, Mh. Chemie, im Druck. 


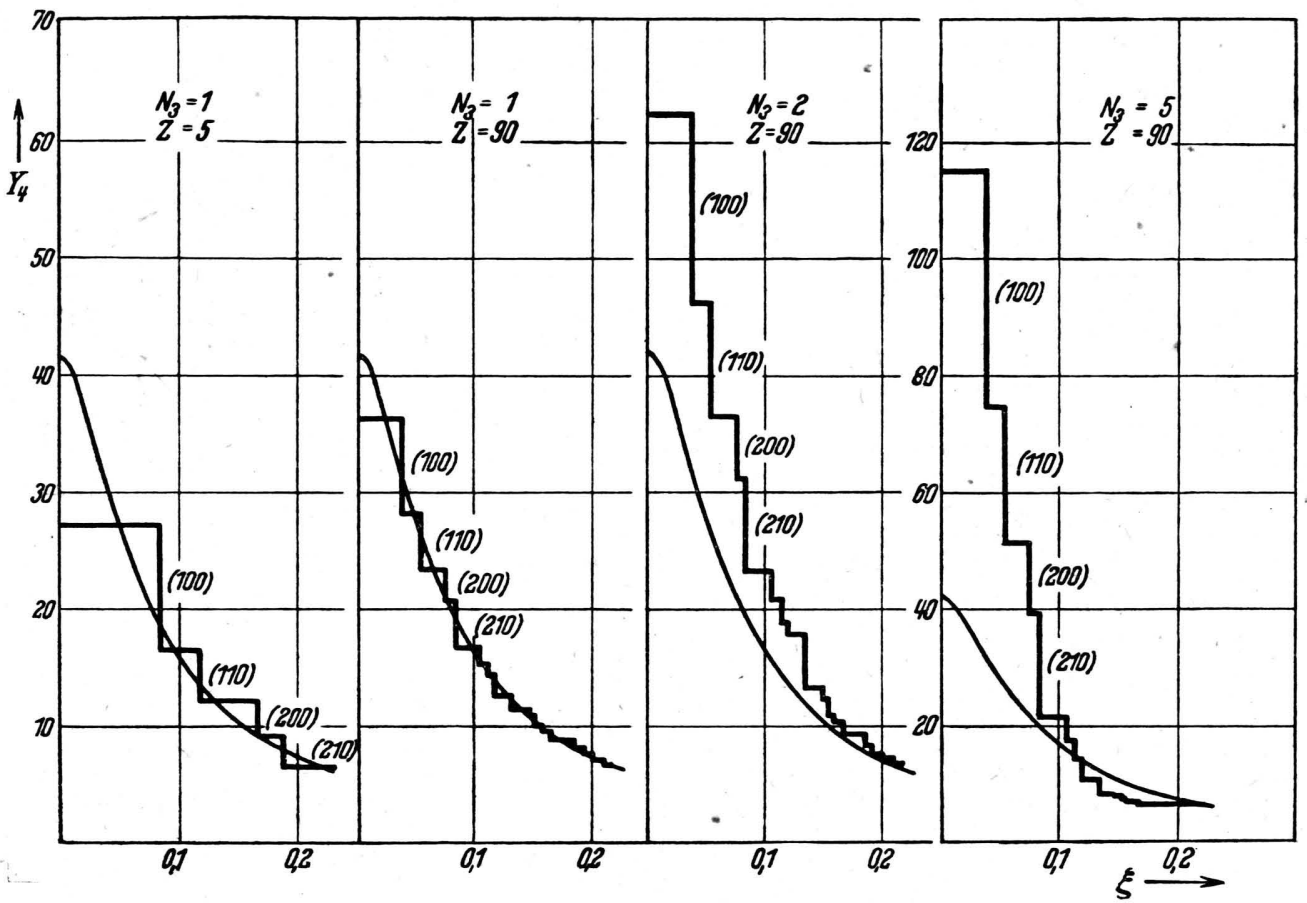

Abb. 4. Elastische Streuabsorption (pro Atom) nicht aufgelöster Kristalle $(a=3 \AA$ A) verschiedener Dicke $\left(N_{3}=1,2,5\right)$ und amorpher Schichten gleicher Belegung.

$$
Y_{4}={ }_{a} N_{e} / N_{0} \varkappa \lambda^{2} Z^{4 / 3} ; \xi=\frac{\sin \vartheta / 2}{\lambda Z^{1 / 3}} ; \varkappa=1,52 \cdot 10^{-18} ; \lambda \text { in } \AA \text {. }
$$

Atome in Richtung der Achse $\mathfrak{a}_{i} ; A_{1}=\frac{a_{1}}{\lambda} \pi \sin \alpha$; $A_{2}=\frac{a_{2}}{\lambda} \pi \sin \beta ; A_{3}=\frac{a_{3}}{\lambda} 2 \pi \sin ^{2} \frac{\vartheta}{2}$ mit den Winkeln $\alpha, \beta, \vartheta$ zwischen der Streurichtung und den Achsen $a_{1}, a_{2}, a_{3}$ und mit der Wellenlänge $\lambda$.

Die Gesamtintensität eines Maximums ergibt sich aus (20) durch Integration über $\alpha$ und $\beta$ zu:

$$
\begin{aligned}
N_{h l_{l} l} & =\iint N_{e}^{\varkappa} d \alpha d \beta \\
& =N_{0} \Phi^{2} S^{2} \frac{\sin ^{2} N_{3} A_{3}}{\sin ^{2} A_{3}} \cdot \frac{\lambda^{2}}{a_{1}^{2} a_{2}^{2}} L_{1} L_{2},
\end{aligned}
$$

mit den Millerschen Indices $(h k l)$ und der Länge $L_{i}=N_{i} a_{i}$ des Gitters in Richtung der Achse $a_{i}$. Voraussetzung war bei der Integration, daß durch hinreichend große $N_{1}$ und $N_{2}$ und kleine $N_{3}$ - also durch flache Kristalltafeln - dafür gesorgt wurde, daß die Funktion $\frac{\sin ^{2} N_{3} A_{3}}{\sin ^{2} A_{3}}$ so langsam veränderlich wurde, daß sie ebenso wie $\Phi^{2} S^{2}$ vor das Integral gezogen werden konnte. Für hin- reichend kleine Werte von $N^{3} A^{3}$ wird (21) angenähert:

$$
N_{h k l}=N_{0} \Phi^{2} S^{2} N_{3}{ }^{2} \frac{\lambda^{2}}{a_{1}^{2} a_{2}^{2}} L_{1} L_{2} .
$$

Kontraste nicht aufgelöster Krist a 11 e. Der Streuabsorptionskontrast ausgedehnter Kristallplatten, deren Gitterstruktur im Mikroskop nicht aufgelöst wird, kommt durch Ausschaltung aller Interferenzstrahlen zwischen $\vartheta=180^{\circ}$ und dem Öffnungswinkel $\vartheta$ des Objektivs durch die Aperturblende zustande. Der Intensitätsverlust ist also durch Summation der Interferenzintensitäten nach Gl. (21) in dem genannten Winkelbereich, unter Berücksichtigung ihrer Häufigkeit, zu bestimmen. Als Beispiel ist in Abb. 4 der so gewonnene Kontrast eines einfachen kubischen Gitters $(S=1)$ mit $a=3 \AA$ aufgetragen.

Hier werden jedoch nur Interferenzen mit Indices kleiner als (600) berücksichtigt. Für größere Streuwinkel wurde die Streuabsorption mit der von ideal ungeordneten Schichten gleicher Be- 
legung (gleiche Atomzahl pro $\mathrm{cm}^{2}$ ) identifiziert, die zum Vergleich in Abb.4 eingezeichnet ist, denn die Interferenzintensität des Kristalls stellt einen Mittelwert des zugehörigen Bereichs der Streuintensität einer amorphen Schicht gleicher Belegung dar. Zwischen beiden besteht um so bessere Übereinstimmung, je geringer die Veränderlichkeit des Atomfaktors ist, also bei großen Streuwinkeln. Als Atomfaktor wurde die für das Thomas-Fermi-Modell von Bethe tabellierte Funktion

$$
\Phi^{2}=\frac{\varkappa}{8 \pi} Z^{2 / 3}\left(\frac{1-f}{\xi^{2}}\right)^{2}
$$

nach graphischer Interpolation eingesetzt.

Mit Verkleinerung der Objektivapertur $\vartheta$ findet durch Ausschaltung weiterer Interferenz-Maxima von der Bilderzeugung eine diskontinuierliche Zunahme des Kontrastes statt, der für einzelne Netzebenen $\left(N_{3}=1\right)$ um den Mittelwert des Kontrastes einer ideal ungeordneten Schicht schwankt. Durch Hintereinanderschalten mehrerer Netzebenen, also im Raumgitter, können bei kleinen Aperturen durch Interferenz der von hintereinander liegenden Atomen stammenden Streuwellen u. U. wesentlich stärkere Kontraste als bei amorphen Objekten gleicher Bedeckung auftreten. Von dieser Verstärkung der Kontraste soll Abb. 4 mit $N_{3}=1$ bzw. 2 und 5 einen Eindruck vermitteln. Solange es sich nicht um mono-atomare Schichten handelt, sind also die durch den Kontrast nachweisbären „Grenzdicken“ im kristallinen $\mathrm{Zu}$ stand dünner als im amorphen.

Selbstverständlich ist immer darauf $\mathrm{zu}$ achten, daß die Voraussetzung der kinematischen Theorie, die die Grundlage der hier abgeleiteten Beziehungen bildet, erfüllt ist. Diese Voraussetzung trifft z. B. auch nicht mehr angenähert für den in Abb. 4 mit $N_{3}=5$ und $Z=90$ angegebenen Fall zu, da dort die Intensität des Primärstrahls schon wesentlich durch die Interferenzintensität beeinflußt wird, denn für $\xi=0$ gilt dort:

$$
\left(\frac{\Delta I}{I_{0}}\right)_{\text {amorph }}=0,35 \text { und }\left(\frac{\Delta I}{I_{0}}\right)_{\text {krist. }}=0,97 \text { ! }
$$

\section{Phasen-Kontrast ${ }^{15}$}

Auch wenn das Auflösungsvermögen des Elektronenmikroskops zur Auflösung der Kristall-

$15 \mathrm{H}$. B o e r sch, eingesandt an Mh. Chemie im Juni 1946. gitterstruktur ausreichen würde, so würde bei ,idealer" Abbildung dennoch die Gitterstruktur zunächst unsichtbar bleiben.

Es handelt sich hier um ein Problem, das Z er nicke ${ }^{16}$ für das Lichtmikroskop am Beispiel des Gegensatzes „Phasen-Amplituden“-Gitter behandelt hat. Das übliche Gitter der Lichtoptik ist ein Amplitudengitter und besteht aus Streifen von abwechselnd verschiedener Durchlässigkeit, die sich deshalb im Bilde durch ihren verschiedenen Kontrast kennzeichnen. Das Phasengitter dagegen besteht aus Streifen von abwechselnd verschiedenem Brechungsindex, die wegen ihrer gleichen Durchlässigkeit im Bilde keine Kontrastdifferenzen gegeneinander aufweisen. In beiden Fällen treten jedoch Beugungsfiguren auf, die sich unter Umständen bei gleichen Intensitätsverhältnissen nur durch ihre Phasenbeziehungen unterscheiden. Um nun auch die Gitterstruktur eines Phasengitters durch Kontraste abzubilden, um also ein Phasengitter in ein Amplitudengitter umzuwandeln, nimmt Zernicke eine Phasenschiebung des Zentralflecks der Beugungsfigur (bei kleinen Phasendifferenzen um $\lambda / 4$ ) vor.

Auch das Kristallgitter ist als Phasengitter für Elektronen aufzufassen, denn die Beugungsfigur kommt nicht durch periodische Absorption im Objekt, sondern durch das periodisch wechselnde Potentialfeld, also durch periodische Anderung des Brechungsindex, zustande. Daher ist zur Kennzeichnung der Kristallgitterstruktur im Elektronenmikroskop, wie im Lichtmikroskop, eine Phasenschiebung des Primärstrahls im Beugungsbild oder v.v. seiner Umgebung mittels einer „Phasenplatte" notwendig (vergl. Abb.5).

Im folgenden sollen nun die Kontraste von Kristallgittern unter Voraussetzung einer ,idealen“ Phasenschiebung bestimmt werden ${ }^{17}$. Es soll also

16 F. Z e r n i cke, Z. techn. Physik 16, 454 [1935].

17 Auch die Bildkontraste von Einzelatomen würden durch Phasenschiebung geändert werden. Da in diesem Fall die Beugungsfigur die Gesamtobjektivöffnung kontinuierlich ausfüllt, muß der gesamte umständliche Apparat der Abbildungstheorie aufgeboten werden, um den Einfluß von kontinuierlichen und diskontinuierlichen Phasenschiebungen auf die Abbildung von Einzelatomen übersehen zu können. Auf jeden Fall würde eine geeignete Phasenschiebung eine Erhöhung der Kontraste gegenüber den allein durch Streuabsorption verursachten Kontrasten des Einzelatoms bringen. Ebenso können auch die Bildkontraste größerer Objekte, deren atomare Struktur nicht aufgelöst wird, durch das angegebene PhasenKontrastverfahren verstärkt werden. 


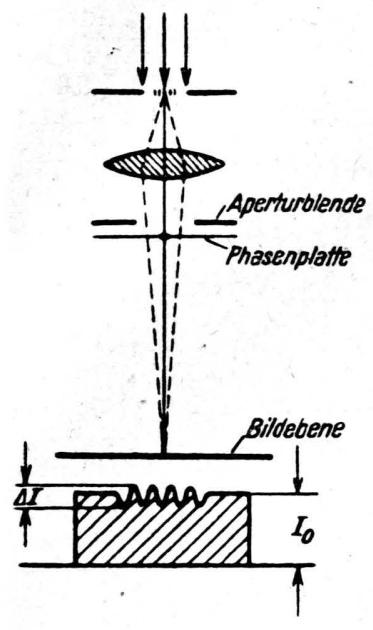

Abb. 5. Phasenkontrastverfahren. vorausgesetzt werden, daß die dem Bilde zugrunde liegende Beugungsfigur durch ein Amplitudengitter erzeugt wird. Die Intensitäten dieser Beugungsfigur stammen jedoch vom Kristallgitter. Es handelt sich demnach um eine optimale Abschätzung.

Ferner möge angenommen werden, daß alle Maxima nullter und erster Ordnung an der Bilderzeugung teilnehmen, daß aber alle Interferenzmaxima höherer Ordnung durch die Aperturblende, z. B. wegen der Linsenfehler, ausgeschaltet werden.

Entsprechend der Fourier-Analyse wird durch diese Einschränkung eine einfache sinusförmige Verteilung der Durchlässigkeit bzw. des Brechungsindex oder des Potentials vorausgesetzt. Unter diesen Umständen gehen jedoch gerade die Feinheiten der Potentialverteilung im Kristall, wie sie in der normalen Struktur- und FourierAnalyse durch Berücksichtigung der höheren Interferenzordnungen bestimmt werden, verloren. Eine Strukturanalyse im bisherigen Sinn ist also durch die Beschränkung auf die Maxima nullter und erster Ordnung ausgeschlossen.

Doch bietet die Abbildung auch unter diesen Umständen den Vorteil, daß die individuelle Gestalt des Kristalls gleichzeitig mit dem Netzwerk des Elementargitters dargestellt wird und daß Kristallbaufehler, also Randverzerrungen, Anlagerungen an die Oberfläche, Fehl- und Lockerstellen und $\mathrm{Z}_{w}$ ischengitterplätze, abgebildet werden. Für diese Fälle geben die bisherigen Methoden der Strukturanalyse nur Durchschnittswerte oder erfassen sie überhaupt nicht. Die Abbildung der Kristallstruktur wäre also auch unter den angegebenen Einschränkungen von grundsätzlichem . Interesse.

a) Intensität der Amplituden-GitterInterferenzen

Zur Bestimmung der Interferenzintensitäten des Amplitudengitters möge angenommen werden, daß es eine mit der Periode $a_{1}$ bzw. $a_{2}$ in Richtung der aufeinander senkrecht stehenden Achsen $a_{1}$ und $a_{2}$ "wechselnde Durchlässigkeit besitzt, die zu einer periodischen Abhängigkeit der Amplitude der durchgehenden Strahlung, unmittelbar hinter dem Gitter, gemäß der Funktion :

$$
u_{D}^{A}=u+\Delta u \cos ^{2} \pi \frac{\xi}{a_{1}} \cos ^{2} \pi \frac{\eta}{a_{2}}
$$

führt. Für kleine Streuwinkel wird die Amplitude der gestreuten Strahlung des Amplitudengitters nach der Kirchhoffschen Formel:

$$
u^{A}=\frac{1}{\lambda} \int_{\left(L_{1}, L_{2}\right)} \int_{D}^{A} e^{i k(\alpha \xi+\beta \eta)} d \xi d \eta .
$$

Aus Gl. (25) ergibt sich die Amplitude der Beugungsfigur:

$$
\begin{aligned}
& u^{A}=\frac{L_{1}}{\lambda} \frac{L_{2}}{\lambda} \frac{\sin N_{1} A_{1}}{N_{1} A_{1}} \frac{\sin N_{2} A_{2}}{N_{2} A_{2}} . \\
& \left(u+\frac{\Delta u}{4} \frac{1}{\left(\frac{a_{1}^{2}}{\lambda^{2}} \sin ^{2} \alpha-1\right)} \frac{1}{\left(\frac{a_{2}^{2}}{\lambda^{2}} \sin ^{2} \beta-1\right)}\right)
\end{aligned}
$$

Durch Quadrierung und Integration nach $\alpha$ und $\beta$ folgt aus (26) die gesamte vom Amplitudengitter in ein Interferenzmaximum gestreute Intensität:

$$
\begin{aligned}
& I_{(00)}^{A}=\left(u+\frac{\Delta u}{4}\right)^{2} L_{1} L_{2}, \\
& I_{(10)}^{A}=\left(\frac{\Delta u}{8}\right)^{2} L_{1} L_{2}, \\
& I_{(11)}^{A}=\left(\frac{\Delta u}{16}\right)^{2} L_{1} L_{2} .
\end{aligned}
$$

Den Voraussetzungen gemäß wird jetzt die Interferenzintensität des Amplitudengitters mit der des Kristallgitters zur Übereinstimmung gebracht. Für geringe prozentuelle Durchlässigkeitsdifferenzen $\frac{\Delta u}{u}$ im Amplitudengitter wird dann:

$$
\begin{aligned}
& N_{0}=I_{(00)}^{A}, \\
& N_{(h 00)}=I_{(10)}^{A} .
\end{aligned}
$$

Nicht berücksichtigt wird die Interferenz (11), die wegen ihrer geringen Intensität für die Bildkontraste als nicht sehr wesentlich erscheint. Zwar hat diese Vernachlässigung ein anderes „Bild“ zur Folge, wie auch frühere Versuche über die „übermikroskopische Abbildung von Kristallgit- 
tern mit dem Lichtmikroskop" ${ }^{18}$ zeigten. Jedoch dürften diese Bildänderungen für die Abschätzung der Kontrastdifferenzen ohne Belang sein.

\section{b) Kontraste a ufgelöster Kristall- git te r}

Aus den Gln. (28) ergeben sich für $\Delta u \ll u$ die Kontraste der Atome in Kristallgittern in einer Abbildung nach dem Phasenkontrastverfahren zu:

$$
\frac{\Delta I}{I_{0}}=2 \frac{\Delta u}{u}=16 \cdot 10^{-8} \Phi S \frac{\lambda}{a_{1} a_{2}} N_{3} .
$$

Das Phasenkontrastverfahren gestattet nach Gl. (29) den Nachweis der Atome in mono-atomaren Kristallschichten schon für Ordnungszahlen $Z \geqq 3$, wenn ein einfach kubisches Gitter $(S=1)$ mit der Gitterkonstante $a=3 \AA$ durch die Wellenlänge $\lambda=$ 0,05 bei einem idealen Auflösungsvermögen von $3 \AA$ abgebildet wird. Die Ein-

führung dieses Verfahrens in die Elektronenmikroskopie bedeutet also einen ganz wesentlichen Fortschritt, da damit die Atome der organischen Verbindungen beobachtbar werden. Mittels elastischer Streuabsorption sind dagegen unter sonst gleichen Bedingungen amorphe Schichten gleicher Bedeckung, deren Atome nicht aufgelöst werden, erst von Ordnungszahlen $Z \geq 138$ nachweisbar. Der Faktor $N_{3}$ in Gl. (29) bedeutet, daß auf der Achse $\mathfrak{a}_{3}$ liegende Atome im Bilde aufeinander projiziert werden.

$$
\text { c) Phasenschiebung }
$$

Damit eine einwandfreie Phasenschiebung vorgenommen werden kann, darf in der Beugungsfigur eine Überdeckung zwischen Primärstrahl und dem Teil der Beugungsfigur, der in der Phase gegenüber dem Primärstrahl verschoben werden soll, nicht stattfinden. Hiermit ist eine Beziehung zwischen der Kondensor-Apertur $\alpha_{k}$ und der Größe $D$ des Objekts, das allenfalls noch mit vol-

18 H. B o e r s c h, Z. techn. Physik 19, 337 [1938]; Physik. Z. 39, 933 [1938]; Z. Elektrochem. 46, 444 [1940]. lem Kontrast abgebildet werden kann, aufgestellt:

$$
a_{k}<\frac{\lambda}{D} \text {. }
$$

Um also möglichst große Objekte mit dem Phasenkontrastverfahren abbilden $\mathrm{zu}$ können, ist eine möglichst parallele Beleuchtung notwendig.

Eine Phasenschiebung ist wegen der Wellenlängenabhängigkeit $\lambda=\sqrt{\frac{150}{U}}$ der Elektronen wellen vom Potential $U$ dadurch möglich, daß die verschiedenen Interferenzstrahlen Wege mit verschiedenem Weg-Potential-Integral durchlaufen. In $\mathrm{Abb}$. (6a, b, c) sind verschiedene Anordnungen dargestellt, die im folgenden diskutiert werden sollen.

Folie. Der Vorschlag (Abb.6a), eine Folie als Phasenplatte zu benutzen, beruht darauf, daß der feste Körper ein konstantes (positives) inneres Potential von der Größenordnung $\Delta U=10$ bis 20 Volt besitzt. Wenn also ein Elektronen-

strahl eine Folie der Stärke $x$ durchsetzt, so ist seine Phase um den Betrag:

$$
\frac{\Delta \lambda}{\lambda}=\frac{1}{300} x \lambda \Delta U
$$

verschoben. Für ein inneres Potential von 10 Volt muß demnach die Folie $150 \AA$ A stark sein, um bei einer Wellenlänge von $0,05 \AA$ eine Phasenschiebung von $90^{\circ} \mathrm{zu}$ erzielen. Damit eine Phasenschiebung des Primärstrahls gegen die übrige Beugungsfigur eintritt, ist die Folie für den Primärstrahl ausgespart.

$\mathrm{Ob}$ die Streuung in der Folie eine wesentliche Beeinträchtigung der Bildqualität mit sich bringt, ist ein Problem, das im folgenden untersucht werden soll. Die Gesamtstreuung in der Phasenfolie. die beispielsweise aus Beryllium bestehen soll, beträgt unter den gegebenen Voraussetzungen schätzungsweise etwa $50 \%$ der Elektronen, die die Folie durchsetzen. (Hiervon entfallen 19\% auf die elastische Gesamtstreuabsorption, während die restlichen $31 \%$ als Anteil der unelastischen Gesamtstreuung geschätzt werden.) Da aber nur die Interferenzmaxima 1. Ordnung die Phasenfolie 
durchsetzen, wird der Bilduntergrund durch die Streuung in der Phasenfolie nur sehr wenig aufgehellt. Um z. B. nach (29) einen Bildkontrast von $\Delta I / I_{0}=0,2$ im Bilde $\mathrm{zu}$ erzeugen, beträgt nach $(27 \mathrm{~b}, 28 \mathrm{~b}, 29)$ die gesamte in die Interferenzmaxima gestreute Intensität

$$
4 I_{(100)} / I_{0}=\frac{1}{64}\left(\frac{\Delta I}{I_{0}}\right)^{2} L_{1} L_{2}=6,2 \cdot 10^{-4}
$$

für den Fall, daß das Objekt die gesamte Objektivöffnung ausfüllt. Da hiervon nur wieder $50 \%$ in das Bild gestreut werden, ist also die so verursachte Aufhellung des Bilduntergrundes zu vernachlässigen. Hierbei ist noch zu berücksichtigen, daß nicht alle in der Plasenplatte gestreuten Elektronen in das Bild, insbesondere von kleinen Objekten, gelangen. Durch die Einschaltung der Folie tritt also nur eine Schwächung der Interferenzintensitäten um etwa $50 \%$ ein, die sich in einer Verminderung der Kontraste um den Faktor $1 / \sqrt{2}$ gegenüber den theoretischen Werten (29) äußert. Schwierigkeiten prinzipieller Natur scheinen also für eine Phasenschiebung durch Folien nicht zu bestehen.

Auf jeden Fall bringt aber die Einschaltung von Folien in den Strahlengang Schwierigkeiten $e x$ perimenteller Natur mit sich, die einerseits durch die mechanische Empfindlichkeit derartig zarter Gebilde, andererseits durch die Bildung und die Aufladung von Niederschlägen auf der Folie bedingt sind, die durch die Elektronenstrahlung selbst verursacht werden.

Elektrostatische Felder. Man wird daher versuchen, elektrostatische Potentialfelder, wie sie z. B. durch die in Abb. 6b, c gezeigten Elektrodenanordnungen erzeugt werden, zur Phasenschiebung heranzuziehen. Diese Anordnungen bestehen aus zwei Elektroden: die äußere befindet sich auf Anodenpotential, die innere auf dem Potential $\Delta U$. Jedoch erscheint die experimentelle Realisierung der in Abb. 6 b gezeigten Anordnung wegen der Geringfügigkeit der geometrischen Dimensionen als wenig aussichtsreich. Außerdem hat sie den Nachteil, daß auch hier der Elektronenstrahl isolierende Niederschläge erzeugen kann, die zu störenden Aufladungen führen können. Diese Nach. teile zeigt die in Abb. 6c wiedergegebene. Anordnung nicht. Damit hier bei möglichst kleiner Linsenwirkung eine möglichst große Phasenschiebung zwischen Zentral- und Randstrahl resultiert, müs- sen die Elektroden dem Randstrahl möglichst stark genähert werden. Die Phasenschiebung ist hier eine Funktion des Durchmessers der Beugungsfigur in der Weise, daß die Phasenschiebung mit der Größe der untersuchten Objekte abnimmt. Um eine Störung der Abbildung durch die Linsenwirkung dieser Anordnung, möglichst herabzusetzen, wird man die Elektrodenpotentiale möglichst klein halten.

Abgesehen von den diskutierten Phasenplatten, wird allein schon die sphärische Aberration des Objektivs oder u. U. eine extrafokale Einstellung zu der notwendigen Phasenschiebung und damit zu einer Abbildung nach dem Phasenkontrastverfahren führen, so daß dieses Verfahren vielleicht jetzt schon bei der Abbildung kleinster Objekte und von Objekträndern eine bisher nicht erkannte Anwendung findet.

\section{Filterung}

Eine andere Möglichkeit, Kontraste zu erzeugen, besteht darin, die Elektronen, die im Objekt Geschwindigkeitsverluste erlitten haben, durch ein Geschwindigkeitsfilter (Abb. 7) auszuscheiden. Hierdurch wird ein Defizit von Elektronen an den Bildstellen erzeugt, an denen im Objekt unelastisch gestreut wird. Eine derartige Filterung erscheint deshalb durchführbar zu sein, weil die Geschwindigkeitsverluste nicht beliebig klein sind, sondern nach den Versuchen von $\mathrm{Fr}$ a $\mathrm{nk}$ und $\mathrm{Her} \mathrm{z}$ am Einzelatom und nach den Versuchen von $R \mathrm{u}$ th e mann am festen Körper in diskreten Stufen von mehr als einigen Volt erfolgen.

Zur Abschätzung der Wirkung eines derartigen Filters ist die Kenntnis der Gesamtzahl der unelastisch gestreuten Elektronen notwendig, die für den Fall des Aluminiums durch die Beziehung (12) dargestellt wird.

a) Kontrast flächen hafter Objekte

Der durch ein Filter erzeugte Elektronen-Kontrast wird für flächenhafte Objekte, deren Atomabstände nicht auf-
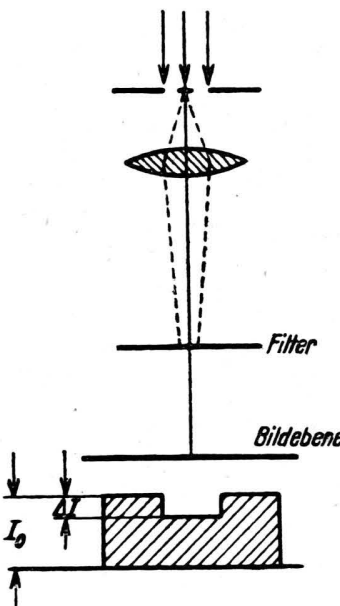

Abb. 7. Kontrast durch Ausfilterung der unelastisch gestreuten Elektronen 
gelöst werden :

$$
\frac{\Delta I}{I_{0}}=n \frac{a^{N_{u}}(0)}{N_{0}} .
$$

Dieser Kontrast stimmt also mit dem für unelastische Streuabsorption im Fall $\xi=0$ überein. Eine Al-Schicht muß bei einem prinzipiellen Auflösungsvermögen von $3 \AA$ und einer Wellenlänge $\lambda=0,05 \AA$ eine Dicke $D \geq 65 \AA$ (im amorphen $\mathrm{Zu}$ stand) besitzen, um mittels der elastischen Streuabsorption nachgewiesen werden zu können. Wenn zusätzlich die unelastisch gestreuten Elektronen weggefiltert werden, so ergibt sich eine nachweisbare Schichtdicke von $D \geq 22 \AA$.

\section{b) Kontrast von Einzelatomen}

Für Einzelatome ist der Elektronenverlust, der durch das Filter bewirkt wird, wieder, wie bei der Streuabsorption, auf das Beugungsscheibchen zu beziehen, als welches das als Punkt gedachte Atom abgebildet wird:

$$
\frac{\Delta I}{I_{0}}=4 \pi 10^{16} Z^{2 / 3} \xi^{2} \frac{{ }_{a} N_{u}(0)}{N_{0}} .
$$

Für den Fall des Al-Atoms ergibt sich demnach ebenfalls eine Kontraststeigerung um den Faktor 3 gegenüber der elastischen Streuabsorption, wenn die unelastische Gesamtstreuung durch ein Filter beseitigt wird.

c) Kontrast aufgelöster Kristall-

$$
\text { git ter }
$$

Für den Fall, daß das Auflösungsvermögen ausreicht, Atomabstände im Kristall zu trennen, soll vorausgesetzt werden, daß die unelastischen Streuvorgänge nicht gleichmäßig über den Kristall verteilt sind, sondern periodisch vom Ort mit den Gitterkonstanten als Grundperioden abhängen. Um zu einer Abschätzung der maximal möglichen Kontraste zu gelangen, soll zusätzlich angenommen werden, daß die Ortsabhängigkeit der inkohärenten Streuung im Kristall durch eine einfache Sinusfunktion ohne konstanten Anteil darstellbar ist. Dann wird die kohärente Erregung unmittelbar hinter dem Gitter durch die Funktion (24) darstellbar. Die unelastische Streuung in Verbindung mit dem Geschwindigkeitsfilter bewirkt also eine Darstellung des Kristallgitters als "Amplitudengitter". Für $\Delta u \ll u$ wird die von die- sem Gitter absorbierte Intensität

$$
I_{a}=\frac{3}{2} u \Delta u L_{1} L_{2}
$$

unter der Voraussetzung, daß die Primärintensität vor dem Gitter

$$
I_{0}=(u+\Delta u)^{2}
$$

beträgt und Glieder der Größenordnung $\Delta u^{2}$ vernachlässigt werden können. Diesem Intensitätsverlust wird die Gesamtintensität der inkohärenten Streuung (Streuabsorption für $\xi=0$ ) gleichgesetzt:

$$
{ }_{a} N_{u}(0)=\frac{3}{2} u \Delta u
$$

Hieraus ergibt sich der maximale Elektronenkontrast der Atome des Kristallgitters, der durch Ausfilterung der unelastischen Streuung entsteht, zu:

$$
\frac{\Delta I}{I_{0}}=2 \frac{\Delta u}{u} \frac{4}{3} \frac{a N_{u}(0)}{N_{0}} \cdot \frac{N_{3}}{a_{1} a_{2}} .
$$

Ein Al-Kristall ( $A 1$-Typ) mit der Gitterkonstante $a=4,04 \AA$, der in Richtung einer Hauptachse mit einer Wellenlänge von $0,05 \AA$ projiziert wird, muß nach (37) unter Berücksichtigung von (12) eine Dicke von $24 \AA$ besitzen, um die Ätome durch ihre Kontraste erkennbar zu machen, während das Phasenkontrastverfahren diese Atome schon in mono-atomarer Schicht hinreichend kennzeichnen könnte.

Durch geeignete Vorzeichenwahl der Phasenschiebung im Phasenkontrastverfahren ist, wie sich aus Symmetriegründen ergibt, der Kontrast des Phasenkontrastverfahrens und des Streuabsorptions- bzw. Filterverfahrens gleichsinnig $\mathrm{zu}$ überlagern.

Die Kontraststeigerung dureh Geschwindigkeitsfilterung dürfte vor allem für leichte Substanzen wirksam sein, deren unelastische Gesamtstreuung die elastische überwiegt, so z. B. für organische Präparate. Andererseits wird die Filterung insbesondere bei dicken Präparaten eine Erhöhung des Auflösungsvermögens mit sich bringen, da das Auflösungsvermögen infolge der Zunahme der unelastischen Streuung mit der Präparatdicke abnimmt. Der Anwendungsbereich des Elektronenmikroskops kann also im Hinblick auf die Abbildung dünnerer und dickerer Objekte und die $\mathrm{Ab}$ bildung bei streifendem Einfall durch geeignete Fil- 
terung erhöht werden. Aber auch der unelastische Untergrund in Elektronenbeugungsdiagrammen kann durch eine geeignete Filterung beseitigt werden, so daß auch hier eine Erweiterung des Anwendungsbereichs auf dickere Präparate und bei streifendem Einfall zu erwarten ist.

\section{Linsenfehler}

Bei der Kontrastbildung durch Streuabsorption verhindert die Aperturblende, daß die gestreuten Elektronen an den zugehörigen Bildort gelangen. In gleicher Weise können auch die Linsenfehler wirken, die also nicht nur eine schädliche Auflösungsverminderung, sondern auch eine im allgemeinen sehr erwünschte Steigerung der Kontraste zur Folge haben können.

Der experimentelle Nachweis der Kontraststeigerung durch Linsenfehler gelang für die sphärische Aberration in besonders eindrucksvoller Weise an Hand der sogenannten "Nebenbilder"19 (Abb. 8). Nebenbilder kommen durch Kristallgitter-Interferenzstrahlen zustande, die infolge des Öffnungsfehlers der Linse nicht an den zugehörigen Bildort gelangen. Sie erzeugen ein Bild „,neben“ dem Primärbild, das durch den Primärstrahl entworfen wird. Die Intensität des Nebenbildes geht also dem Primärbild verloren.

Auf die kontraststeigernde Wirkung des chromatischen Fehlers hat Hillie ${ }^{20}$ aufmerksam gemacht. G a b o r ${ }^{21}$ weist betont auf diese Effekte hin, die für die praktische Mikroskopie von besonderem Interesse sind, da dort meistens nicht die optimalen Objektivaperturen verwendet werden, sondern erheblich höhere.

Der Durchmesser des Öffnungsfehlerscheibchens in der Bildebene beträgt:

$$
d_{\ddot{o}}=2 C_{\ddot{o}} \vartheta^{3},
$$

der des chromatischen Fehlerscheibchens:

$$
d_{\mathrm{F}}=2 C_{\mathrm{F}} \vartheta \frac{\Delta U}{U}
$$

mit der Öffnungsfehlerkonstante $C_{\ddot{O}}$ und der Farbfehlerkonstante $C_{\mathrm{F}}$, Auf Kreisflächen dieses Durchmessers werden die gestreuten Elektronen ausgebreitet, die nicht von der Aperturblende abgefangen werden. Solange also däs Bild

19 H. B o e r s c h, Z. Physik 121, 745 [1943].

so J. H ill i e r, Canad. J. Res. 17 A, 64 [1939]. des Objekts kleiner ist als das Fehlerscheibchen, tritt nach der schematischen Darstellung Abb.9 eine Erhöhung des Kontrastes ein.

Die eigenartige Erscheinung, daß außer dem scharfen Bild des Objekts das Öffnungsfehlerscheibchen zu beobachten ist, kann darauf zurückgeführt werden, daß die Beugungsfigur einmal durch Streuvorgänge an den Atomen oder den Atomabständen, zweitens durch Streuvorgänge an der „makroskopischen“ Ausdehnung des Streukörpers verursacht wird. Die makroskopische Ausdehung des Streukörpers ergibt eine Beu-

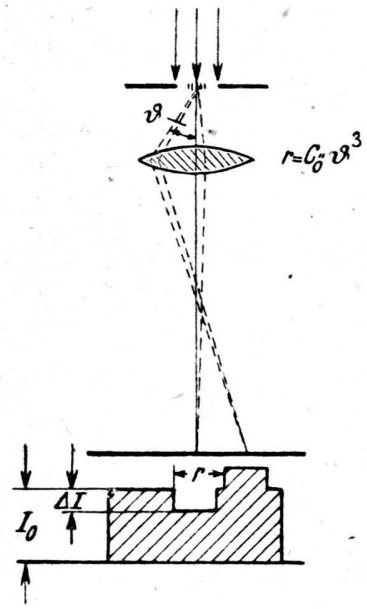

Abb. 8. Entstehung der Nebenbilder.

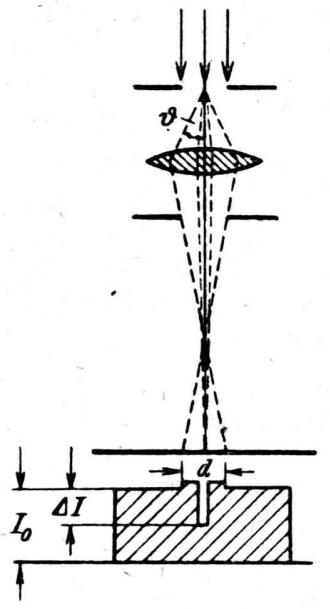

Abb. 9. Kontraste durch Linsenfehler. gungsfigur, deren Breite umgekehrt proportional der Ausdehnung des Streukörpers ist. Diese Beugungsfigur füllt nur den zentralen Teil der freien Apertur des Objektivs aus und führt deswegen zu einer „scharfen“ Abbildung der makroskopischen Figur des Streukörpers. Dagegen haben die kurzen Atomabstände bzw. die Atome selbst eine Beugungsfigur großer Öffnung zur Folge, die die freie Apertur des Objektivs ausleuchtet und daher zu Öffnungsfehlerscheibchen großen Durchmessers führt. Bei Übergang zu kleineren Objekten - also zu Atomen - gehen die erwähnten Beugungsfiguren ineinander über. Ähnlich liegen die Verhältnisse bei dem chromatischen Fehler! Die eigentliche Abbildung des Objekts wird auch hier durch eine Beugungsfigur (aus elastisch gestreuten Elektronen) geringer öffnung vermittelt,

${ }^{21}$ D. G a b o r, The Electron Microscope, London 1945 . 
während die unelastische Streuung diesem Bild das chromatische Fehlerscheibchen überlagert.

Die Intensitätsverteilung im Linsenfehlerscheibchen ist in Abb. 9 nur schematisch angedeutet. Sie ist selbstverständlich eine Funktion der Streuverteilung und bedarf zu ihrer Festlegung eingehender wellenoptischer Überlegungen. Im Extremfall, kleiner Bilddurchmesser und großer Fehlerscheibchendurchmesser, werden die so verursachten Kontraste übereinstimmen mit den Kontrasten der Streuabsorption für den Fall $\xi=0$.

Es möge aber hier betont werden, daß diese Kontraste eine Funktion der Größe des Objekts sind, daß bei größeren Objekten besondere Schwärzungsverteilungen am Rande auftreten, die keine Darstellung der Massendichte-Verteilung des Objekts darstellen, und daß insbesondere bei kleinen Objekten wegen der erwähnten Eigenart der Beugungsfiguren die Linsenfehlerscheibchen sich dem Bild des Objekts so überlagern, daß eine unscharfe Abbildung resultiert. Aus diesen Gründen wird man für extreme Auflösungen versuchen müssen, übersichtlichere Verhältnisse zu schaffen. Dies kann einerseits durch Verwendung möglichst kleiner Objektivaperturen geschehen, die man insbesondere im Hinblick auf die optimale Überlagerung des Öffnungs- und des chromatischen und des Beugungsfehlers dimensioniert, andererseits durch Einschaltung von Elektronenfiltern, die den Einfluß des chromatischen Linsenfehlers auszuschalten versprechen.

\section{Dunkelfeld}

Die bisher untersuchten kontrastbildenden Methoden stellten ausschließlich Hellfeldmethoden dar. Die Dunkelfeldmethode (Abb. 10) wurde
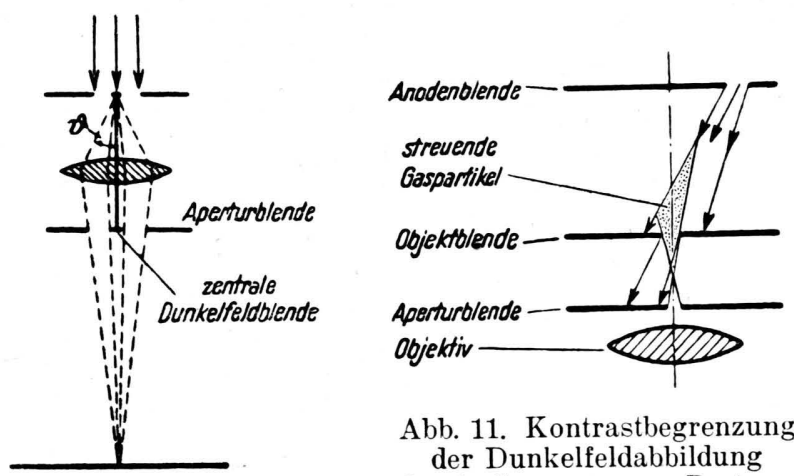

Abb. 11. Kontrastbegrenzung der Dunkelfeldabbildung durch Streuung am Restgas.

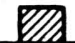

Abb. 10. Dunkelfeldmethode. durch den Verfasser in die Elektronenmikroskopie eingeführt ${ }^{22}$.

Die Dunkelfeldkontraste werden ebenfalls durch gestreute Elektronen verursacht. Eine Kontrastbegrenzung tritt durch Aufhellung des Untergrundes ein, der bei freitragenden Objekten durch Streuung der Primärelektronen an den Gaspartikeln des Restgases (vgl. Abb.11), bei nicht freitragenden Objekten vor allem durch Streuung am Objektträger verursacht wird. Damit der Untergrund möglichst wenig intensiv wird, müssen also die Streumöglichkeiten an objektfremden Partikeln möglichst gering gehalten werden.

\section{a) Nicht freitragende Objekte}

Bei nicht freitragenden Objekten werden die Kontraste durch die Streuung am Objektträger begrenzt. Es sind also Objektträger möglichst geringen Streuvermögens und geringer Dicke auszuwählen. Hierfür kommen - wie bei der Hellfeldmethode - fast nur Folien aus organischem Material (Zaponlack, Kollodium usw.) in Frage. Die geringste herstellbare Dicke dieser Folien dürfte für diese $\mathrm{Zwecke}$ etwa $100 \AA$ betragen.

Als leicht diskutierbares Beispiel für den Elektronenkontrast im Dunkelfeld sei die Anordnung der Abb.10 gewählt, bei der allein die streng parallele Primärstrahlung mit Hilfe einer Blende in der hinteren Brennebene ausgeblendet wird. Im Experiment wird man selbstverständlich andere, leichter realisierbare Anordnungen, z. B. die schiefe, einseitige Dunkelfeldbeleuchtung der Abb. 11, wählen. In der Anordnung nach Abb. 10 ist die Zahl der pro Atom in die Objektivöffnung gestreuten Elektronen

$$
N_{D}={ }_{a} N(0)-{ }_{a} N(\xi) .
$$

Kontrast flächenhafter Objekte. Der Kontrast zweier Schichten aus $n_{1}$ Atomen $/ \mathrm{cm}^{2}$ der Ordnungszahl $Z_{1}$ und $n_{2}$ Atomen $/ \mathrm{cm}^{2}$ der Ordnungszahl $Z_{2}$, deren Atomabstände nicht aufgelöst werden, ergibt sich dann entsprechend. Gl. (19) zu:

$$
\frac{\Delta I}{I}=\frac{n_{1} N_{D}\left(Z_{1}\right)}{n_{2} N_{D}\left(Z_{2}\right)}
$$

für den Fall, daß $\Delta I \ll I$ ist. Nach dieser Beziehung gelingt der Nachweis einer mono-atomaren (amorphen) Schicht für Ordnungszahlen $Z=\mathbf{4 0}$

22 H. B oersch, Ann. Physik 27, 75 [1936]. 
auf einer Zaponfolie von $100 \AA$ Dicke bei einem " prinzipiellen Auflösungsvermögen $d=3 \AA$, wenn vorausgesetzt wird, daß die Atome in der monoatomaren Schicht einen Abstand von $3 \AA$ aufweisen. Hierbei wurde nur die elastische Streuung berücksichtigt, da über die unelastische hinreichende Unterlagen nicht vorhanden sind. Im Experiment dürften diese Verhältnisse durch Einführung eines Geschwindigkeitsfilters zu realisieren sein.

Kontrast von Einzelat omen. DerKontrast von Einzelatomen der Ordnungszahl $Z_{1}$, die in der Gegenstandsebene weit auseinander liegen und die durch ihre Beugungsfehlerscheibchen dargestellt werden, ergibt sich nach den Beziehungen $(16,17,18,41)$ aus der Elektronendichte im Zentrum des Beugungsfehlerscheibchens:

$$
N\left(0, Z_{1}\right)=4 \pi 10^{16} \xi^{2} Z_{1}^{2 / 3}\left({ }_{a} N(0)-{ }_{a} N(\xi)\right)
$$

and aus der Elektronendichte, die durch den $\mathrm{Ob}$ jektträger $\left(n_{2} Z_{2}\right)$, dessen atomarer Aufbau nicht erkennbar sein soll, verursacht wird, $\mathrm{zu}$ :

$$
\frac{\Delta I}{I}=\frac{N\left(0, Z_{1}\right)}{n_{2} N_{D}\left(Z_{2}\right)}
$$

Die Elektronendichte im Zentrum des Beugungsfehlerscheibchens ist in Abb. 12 dargestellt. Unter der Voraussetzung, daß als Objektträger $100 \AA$ starke Zaponfolien dienen, werden bei einem idealen Auflösungsvermögen von $d=3 \AA$ Einzelatome schon von Ordnungszahlen $Z \geqq 13$ an durch ihren Kontrast nachweisbar. Um eine Steigerung der

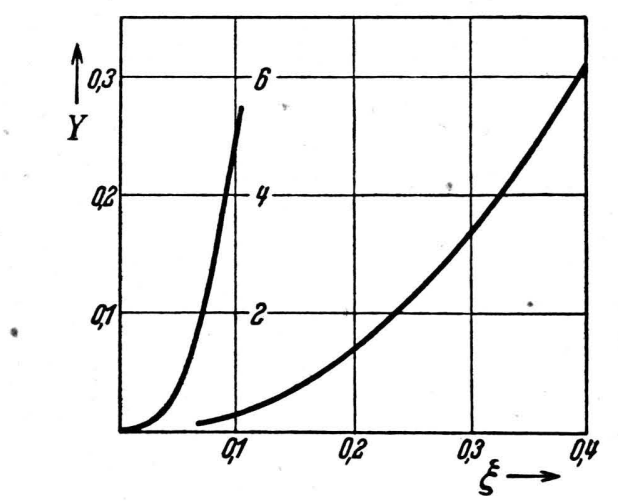

Abb. 12. Elektronendichte im Dunkelfeldbild des Einzelatoms.

$$
\begin{gathered}
Y={ }_{D} N_{e} / N_{0} 4 \pi 10^{16} \varkappa \lambda^{2} Z^{2} ; \xi=\frac{\sin \vartheta / 2}{\lambda Z^{1 / 3}} ; \\
x=1,52 \cdot 10^{-18} ; \lambda \text { in } \AA .
\end{gathered}
$$

Kontraste zu erzielen, ist die Entwicklung schwächer streuender Objektträger erforderlich.

\section{b) Freitragende Objekte}

Bei freitragenden Objekten können die Kontraste durch Herabsetzung des Dampfdrucks in der Nähe des Objekts und durch geeignete Ausblendung der Beleuchtung (vgl. Abb. 11) gesteigert werden. Eine einfache Abschätzung der Zahl der Gasatome, die in das Objekt und in die Objektivöffnung streuen, zeigt, daß bei den üblichen Dampfdrucken $\left(10^{-4} \mathrm{~mm} \mathrm{Hg}\right)$ schon' einzelne Wasserstoffatome als Objekt hinreichende Kontraste zu ihrer Kennzeichnung geben.

Im Experiment ${ }^{23}$ gelang es, $\mathrm{CCl}_{4}$-Dampfstrahlen, die aus einer Kanüle von 0,3 mm Durchmesser und $5 \mathrm{~mm}$ Länge aus einem Vorratsgefäß mit einem Dampfdruck von 0,3 mm in das Vakuum des Mikroskops strömten, elektronenmikroskopisch mittels des Dunkelfeldverfahrens nachzuwe:sen. Unter der gewiß unzutreffenden Annahme, daß in der Kanüle kein Abfall des Dampfdruckes eintritt, wird die Zahl der Chlor-Atome an der Mündung der Düse etwa $15^{15}$ pro $\mathrm{cm}^{2}$. Da der Atomabstand im festen Körper etwa $3 \AA$ beträgt und Schichten mono-atomarer Dicke daher etwa $10^{15}$ Atome enthalten, sind mit diesem Experiment sicher schon Schichten mono-atomarer Dicke aus Chlor-Atomen $(Z=17)$ nachgewiesen. Hierbei ist der Druckabfall in der Düse nicht berücksichtigt; auch ist die Dimensionierung der experimentellen Anordnung hinsichtlich der Streuung an objektfremden Streukörpern noch wesentlich verbesserungsfähig.

\section{c) Dunkelfeld - Hellfeld}

Falls bei hinreichender Auflösung Atome des Kristallgitters mittels der Dunkelfeldmethode abgebildet werden, so treten hier nicht die gleichen Schwierigkeiten auf, die bei der Hellfeldmethode die Einführung des Phasenkontrastverfahrens notwendig machen. Im Beugungsbild des zentrosymmetrischen Phasengitters ist der Primärstrahl um $90^{\circ}$ gegen die Reflexe höherer Ordnung phasenverschoben, die unter sich jedoch höchstens Phasendifferenzen von $\pm 180^{\circ}$ gegeneinander aufweisen. Da im Dunkelfeldverfahren nur Interferenzen höherer als der nullten Ordnung an der Bilderzeugung teilnehmen, ist dort also ein Pha-

${ }_{23}$ H. B o ersch, Z. Physik 107, 493 [1937]. 
senkontrastverfahren nicht notwendig. Da die benachbarten Interferenzen im Beugungsbild des einfach kubischen Gitters keine größenordnungsmäßigen Intensitätsunterschiede gegeneinander aufweisen, werden die entstehenden Gitterbilder fast vollständig durchmoduliert sein, so daß hier etwa dieselben Kontrastgrenzen wie für nicht aufgelöste Atomabstände auftreten. Mit der Dunkelfeldmethode sind also sogar in freitragenden monoatomaren Wasserstoff-Kristallgittern die Atome nachweisbar.

Die Schuierigkeiten der Dunkelfeldmethode liegen auf anderem Gebiet. Sie werden einerseits durch die geringe Bildhelligkeit verursacht, die wegen der Temperaturempfindlichkeit der Objekte nicht durch größere Bestrahlungsintensität kompensiert werden kann. Z.B. ist die Elektronendichte im Dunkelfeldbild des Einzelatoms $(Z=10$, $\lambda=0,05 \AA$, ideales Auflösungsvermögen $3 \AA$ ) um den Faktor $5 \cdot 10^{-5}$ geringer als die Elektronendichte des Primärstrahls. Hierdurch wird die Entwicklung einer besseren Blind-Aufnahmetechnik mit längeren Belichtungszeiten bei Konstanthaltung der Spannungen und der Objektlage notwendig sowie die Entwicklung einer Methode zum Wechsel von Hell- und Dunkelfeldbetrieb ohne Änderung der Scharfeinstellung. Die Schwierigkeit der Dunkelfeldmethode liegt andererseits darin, daß die Aperturblende im Gegensatz zum Hellfeldbetrieb angenähert gleichmäßig ausgeleuchtet wird. Hierdurch werden Aufladungserscheinungen von schlecht leitenden Niederschlägen, die auf der Aperturblende durch die Elektronenstrahlung selbst gebildet werden ${ }^{24}$, für Bildstörungen besonders wirksam. Außerdem muß ja der Durchmesser der Aperturblende den optimalen Auflösungsverhältnissen entsprechend kleingehalten werden. Im Hellfeld können bekanntlich größere Aperturen benutzt werden, als der optimalen Überlagerung der Linsen- und Beugungsfehlerscheibchen entspricht, wodurch Aufladungserscheinungen seltener und nicht so wirksam werden. Trotz dieser Umstände wurde zu einem Zeitpunkt, als das Auflösungsvermögen des elektrostatischen Úbermikroskops im Hellfeld 60 Å betrug ${ }^{25}$, im Dunkelfeldbetrieb ${ }^{24}$ ein Auflösungsvermögen von $85 \AA$ erzielt.

24 H. B o e rsch, Physik. Z. 44, 32 [1943].

25 H. B o ers ch, Physik. Z. 44, 202 [1943]. Inzwischen (Nov. 1944) wurde vom Verfasser das Auflösungsvermögen des elektrostatischen Elektronenmikroskops im Hellfeld auf 28 Â verbessert.
Bemerkenswert ist, daß die Kontraste im Dunkelfeld bei gleichem prinzipiellem und bei gleichem idealem Auflösungsvermögen von der Wellen̉länge unabhängig sind, im Gegensatz zu den Hellfeldmethoden, in denen immer eine Zunahme des Kontrastes mit der Wellenlänge zu konstatieren ist.

Auch differieren im Dunkelfeld die Kontraste von Atomen verschiedener Ordnungszahl nicht so stark wie in Hellfeld-Abbildungen. Der Grund ist darin zu suchen, daß im Dunkelfeld die von $Z$ abhängigen Faktoren im Gegensatz zum Hellfeld gegenläufigen Charakter haben.

\section{Zusammenfassung}

1. Die übliche Kontrastbildung durch Streuabsorption wird durch gestreute Elektronen verursacht, die von der Aperturblende des Objektivs abgefangen werden.

2. Es wird die elastische und die unelastische Streuabsorption des Thomas-Fermi-Atoms bestimmt. Die elastische Streuabsorption kann bis zu den kleinsten Objektivaperturen verfolgt werden. Dagegen wird die unelastische Streuabsorption des Thomas-Fermi-Atoms bei kleinen Aperturen so ungenau, daß sie dort nicht bestimmt werden kann. In dem untersuchten Bereich ist die elastische Streuabsorption etwa um den Faktor $Z$ größer als die unelastische.

3. Die unelastische Gesamtstreuung des Aluminiums kann durch Kombination des Wi d d ing t on schen Gesetzes über den mittleren Energieverlust mit dem $\mathrm{Ruth}$ emannschen Ergebnis über diskrete Energieverluste bestimmt werden.

4. Die Sonderfälle des Wasserstoffs und des Aluminiums zeigen, daß die unelastische Streuabsorption bei kleinen Streuwinkeln außerordentlich stark anwächst, daß sie für geringe Ordnungszahlen die elastische Gesamtstreuung weit überwiegt und daß dieses Verhältnis mit steigender Ordnungszahl abnimmt.

5. Die Streuabsorptionskontraste von Einzelatomen werden auf das Beugungsfehlerscheibchen eines ,punktförmigen“ Objekts bezogen. Sie wachsen mit der Ordnungszahl, dem Auflösungsvermögen und der Wellenlänge. Bei einem Auflösungsvermögen von $3 \AA$ A und einer Wellenlänge von $0,05 \AA$ A treten hinreichende Kontraste für Ordnungszahlen $Z \geqq 64$ auf.

6. Mit Hilfe der Streuabsorption des ThomasFermi-Atoms wird für den Fall der Einzelstreuung 
der Kontrast flächenhafter Objekte bestimmt, deren Atomabstände nicht aufgelöst werden und deren Atome sich in ihren Streuvorgängen gegenseitig nicht beeinflussen. Für Zaponfolien ergibt sich hinreichende Übereinstimmung mit der Erfahrung.

7. Der Streuabsorptionskontrast dünner Kristallschichten, deren Atomabstände nicht aufgelöst werden, deren Atome sich aber in ihrem Streuvermögen gegenseitig beeinflussen (KristallgitterInterferenzen), nimmt wegen der scharfen Kristallgitterinterferenzen sprungweise mit der Verminderung der Apertur des Objektivs zu. Im allgemeinen sind die Kontraste von Kristallgittern stärker als die von amorphen Schichten gleicher Bedeckung, ausgenommen Kreuzgitter.

8. Kontrastbildung durch Phasenkontrastverfahren. In der bisherigen Interferenztheorie wird das Kristallgitter als Phasengitter behandelt. Auch bei elektronenmikroskopischer Abbildung hinreichender Auflösung müßten daher die Atome eines derartigen Gitters unsichtbar bleiben. Erst durch eine Phasenschiebung um $\lambda / 4$ innerhalb der Beugungsfigur könnten die einzelnen Atome durch ihre Kontraste unterschieden werden. Eine derartige Phasenschiebung dürfte im Elektronenmikroskop durch dünne Folien mit einem inneren Potential oder durch elektrostatische Felder, deren Linseneigenschaften zu vernachlässigen sind, realisierbar sein.

9. Aus dem Vergleich der Interferenzintensitäten eines Amplituden- und des Kristallphasengitters ergibt sich, daß die Atome im Kristallgitter dứch das Phasenkontrast-Verfahren bei einer. Wellenlänge von $0,05 \AA$ und einem Auflösungsvermögen von $3 \AA$ A schon von Ordnungszahlen $Z \geqq 3$ nachweisbar sind.

10. Kontrastbildung durch Geschwindigkeitsfilterung. Es wird vorgeschlagen, mittels eines Geschwindigkeitsfilters die unelastisch gestreuten Elektronen aus dem Strahlengang auszusondern! Hierdurch tritt z.B. bei amorphen Aluminiumfolien, deren Atomabstände nicht aufgelöst werden, und bei Einzelatomen eine Verstärkung der Kontraste um etwa den Faktor 3 ein. Besonders wirksam dürften die Geschwindigkeitsfilter für die Kontrastverstärkung leichter Atome sein.
11. Unter der Voraussetzung, daß die unelastische Streuung eine periodische Funktion des Ortes im Kristall ist, wird auch die kohärente Intencität unmittelbar hinter dem Kristall eine periodische Funktion des Ortes. Durch entsprechende Geschwindigkeitsfilterung können also auch die Atome im Kristallgitter durch ihren Kontrast gekennzeichnet werden. Bei einem Aluminiumkristall sollte die Projektion der Atome unter diesen Umständen von einer Kristalldicke von $24 \AA$ an nachweisbar sein.

12. Kontrastbildung durch Linsenfehler kommt dadurch zustande, daß die gestreuten Elektronen nicht an den zugehörigen Bildort gelangen, so daß dort bei hinreichend kleinen Objekten ein Defizit an Elektronen eintritt. Die Linsenfehler wirken also nicht nur auflösungsbegrenzend, sondern auch kontrastverstärkend. Es kann jedoch zweckmäßig sein, übersichtliche und klare Verhältnisse zu schaffen und den Einfluß der Linsenfehler durch optimale Aperturbegrenzung und Filterung möglichst weitgehend auszuschalten.

13. Der Kontrast in der Dunkelfeldmethode wird durch das Verhältnis der Streuung am Objekt und der Streuung an objektfremden Atomen (Objektträger, Restgas) bedingt. In dem untersuchten Beispiel ergibt sich die Streuung aus der Differenz Gesamtstreuung - Streuabsorption.

14. Nicht freitragende Objekte, die auf einer Zaponfolie von $100 \AA$ A Dicke aufgebracht sind, können als Einzelatome bei einem idealen Auflösungsvermögen von $3 \AA$ A von einer Ordnungszahl $Z>13$ an und als mono-atomare amorphe Schichten, deren Atomabstände nicht aufgelöst werden, von einer Ordnungszahl $Z>40$ an nachgewiesen werden.

15. In freitragenden Objekten sind einzelne Wasserstoffatome durch ihren Kontrast nachweisbar.

16. Bei konstanter prinzipieller Auflösung nimmt der Kontrast durch Streuabsorption und durch Filterung proportional dem Quadrat der Wellenlänge, durch das Phasenkontrastverfahren proportional der Wellenlänge zu. Dagegen ist der Kontrast im Dunkelfeld unabhängig von der Wellenlänge. 